\title{
Systematic Review \\ Maternal Bochdalek Hernia during Pregnancy: A Systematic Review of Case Reports
}

\author{
Jin-Young Choi ${ }^{1,+}$, Song-Soo Yang ${ }^{2,+}{ }^{(\mathbb{C}}$, Jong-Hwa Lee ${ }^{3}$, Hyun-Jin Roh ${ }^{1}$, Jun-Woo Ahn ${ }^{1}$, Jeong-Sook Kim ${ }^{1}{ }^{1}$, \\ Soo-Jeong Lee ${ }^{1}$ and Sang-Hun Lee ${ }^{1, * \mathbb{C}}$
}

1 Department of Obstetrics and Gynecology, Ulsan University Hospital, University of Ulsan College of Medicine, Ulsan 44033, Korea; 0734208@uuh.ulsan.kr (J.-Y.C.); 0729345@uuh.ulsan.kr (H.-J.R.); ahnjwoo@uuh.ulsan.kr (J.-W.A.); jeongsookkim@uuh.ulsan.kr (J.-S.K.); exsjlee@uuh.ulsan.kr (S.-J.L.)

2 Department of Surgery, Ulsan University Hospital, University of Ulsan College of Medicine, Ulsan 44033, Korea; ssyang@uuh.ulsan.kr

3 Department of Radiology, Ulsan University Hospital, University of Ulsan College of Medicine, Ulsan 44033, Korea; jhlee@uuh.ulsan.kr

* Correspondence: shlee73@uuh.ulsan.kr; Tel.: +82-052-250-8786

+ These two authors contributed equally as first authors.

check for

updates

Citation: Choi, J.-Y.; Yang, S.-S.; Lee, J.-H.; Roh, H.-J.; Ahn, J.-W.; Kim, J.-S.; Lee, S.-J.; Lee, S.-H. Maternal

Bochdalek Hernia during Pregnancy: A Systematic Review of Case Reports. Diagnostics 2021, 11, 1261. https:/ / doi.org/10.3390/diagnostics11071261

Academic Editor: Antonio

Simone Laganà

Received: 28 June 2021

Accepted: 12 July 2021

Published: 14 July 2021

Publisher's Note: MDPI stays neutral with regard to jurisdictional claims in published maps and institutional affiliations.

Copyright: (c) 2021 by the authors. Licensee MDPI, Basel, Switzerland. This article is an open access article distributed under the terms and conditions of the Creative Commons Attribution (CC BY) license (https:// creativecommons.org/licenses/by/ $4.0 /)$.
Abstract: Background: Since the first report of a diaphragmatic hernia from Ambroise Paré's necropsy in 1610, the Bochdalek hernia (BH) of the congenital diaphragmatic hernia (CDH) has been the most common types with high morbidity and mortality in the neonatal period. Due to the nature of the disease, $\mathrm{CDH}$ associated with pregnancy is too infrequent to warrant reporting in the literature. Mortality of obstruction or strangulation is mostly due to failure to diagnose symptoms early. Data sources and study selection: A systematic literature search of maternal BH during pregnancy was conducted using the electronic databases (PubMed and EMBASE) from January 1941 to December 2020. Because of the rarity of the disease, this review included all primary studies, including case reports or case series that reported at least one case of maternal $\mathrm{BH}$ in pregnant. Searches, paper selection, and data extraction were conducted in duplicate. The analysis was performed narratively regardless of the control groups' presence due to their rarity. Results: The search retrieved 3450 papers, 94 of which were deemed eligible and led to a total of 43 cases. Results of treatment showed 16 cases in delayed delivery after hernia surgery, 10 cases in simultaneous delivery with hernia surgery, 3 cases in non-surgical treatment, and 14 cases in hernia surgery after delivery. Of 16 cases with delayed delivery after hernia surgery, $13(81 \%)$ cases had emergency surgery and three $(19 \%)$ cases had surgery after expectant management. Meanwhile, 10 cases underwent simultaneous delivery with hernia surgery, 6 cases (60\%) had emergent surgery, and 4 cases $(40 \%)$ had delayed hernia surgery after expectant management. 3 cases underwent non-surgical treatment. In this review, the maternal death rate and fetal/neonatal loss rate from maternal $\mathrm{BH}$ was $5 \%(2 / 43)$ and $16 \%(7 / 43)$, respectively. The preterm birth rate has been reported in 35\% (15/43) of maternal $\mathrm{BH}$, resulting from maternal deaths in $13 \%(2 / 15)$ of cases and 6 fetal loss in $40 \%(6 / 15)$ of cases; $44 \%(19 / 43)$ of cases demonstrated signs of bowel obstruction, ischemia, or perforation of strangulated viscera in the operative field, resulting from maternal deaths in $11 \%(2 / 19)$ of cases and fetal-neonatal loss in $21 \%(4 / 19)$ of cases. Conclusion: Early diagnosis and surgical intervention are imperative, as a gangrenous or non-viable bowel resection significantly increases mortality. Therefore, multidisciplinary care should be required in maternal $\mathrm{BH}$ during pregnancies that undergo surgically repair, and individualized care allow for optimal results for the mother and fetus.

Keywords: Bochdalek hernia; diaphragmatic; congenital; pregnancy complication

\section{Introduction}

A diaphragmatic hernia is the protrusion of abdominal organs into the thorax. Since three cases of diaphragmatic hernia, including one traumatic origin, were first reported 
by Ambroise Paré's necropsy description in 1610, George McCauley in 1754 reported the natural course of the disease and gross anatomy of a congenital diaphragmatic hernia $(\mathrm{CDH})$, which related to the rapid death of a neonatal infant through the neonatal necropsy. Interestingly, in his necropsy description in the Philosophical Transactions of the Royal College of Physicians, the herniated organ, including the intestines, spleen, and part of the pancreas, was described on the right side of the infant.

The $\mathrm{CDH}$ has a prevalence of $0.8-5 / 10,000$ in the neonatal period. The Bochdalek hernia $(\mathrm{BH})$ is the most common type of $\mathrm{CDH}$. Surgical treatment is considered the best management for $\mathrm{CDH}$ [1], non-surgical treatment is futile. Carl Hedblom [2] in 1925 surveyed 375 diaphragmatic hernias of varied types, including 44 congenital hernias. In 44 newborns studied, no surgery was performed. Mortality was $75 \%$ in 44 cases with congenital hernias.

Neonatal mortality of $\mathrm{CDH}$ is thought of as life-threatening, as mentioned above. However, a Bochdalek hernia related to $\mathrm{CDH}$ may rarely remain symptomless until adult. Mullins and coworkers [3] reported the incidence of asymptomatic Bochdaleck hernia in the adult is $0.17 \%$ in 22 patients ( 17 women, 5 men) based on 13,138 cases. Brown and his colleagues demonstrated pregnancy was a causative factor of symptomatic Bochdaleck hernia in an adult.

Published articles of $\mathrm{BH}$ presenting in adults due to the rare disease entity are presented as case reports and small series. Therefore, a Bochdaleck hernia during pregnancy is too infrequent to warrant reporting in the literature. Thompson and Le Blanc [4] in 1945 carried out the first successful surgical intervention to treat a congenital diaphragmatic hernia complicating pregnancy and followed a second similar case by Person and his coworker [5] in 1950.

Some authors argue that pregnancy was also overrepresented in cases requiring emergency. However, although requiring immediate treatment regardless of the gestational age because obstruction and gangrene of the herniated viscera affect the survival of mother and fetus, there is still controversy over determining the appropriate timing of treatment for maternal Bochdaleck hernia during pregnancy

We performed a systematic literature review to provide treatment guideline about a maternal Bochdaleck hernia during pregnancy.

\section{Materials and Methods}

\subsection{Sources}

To find articles published in all languages from January 1941 to December 2020, we searched the PubMed (all fields) and the EMBASE databases concerning maternal diaphragmatic hernia during pregnancy using the following search strategies: ("hernia, diaphragmatic" (Medical Subject Headings (MeSH Terms)) ("hernia" AND "diaphragmatic") OR "diaphragmatic hernia" OR ("diaphragmatic" AND "hernia")) AND ("pregnancy" (MeSH Terms) OR "pregnancy" OR "pregnancies") for PubMed, and ("diaphragm" / exp OR diaphragm) AND ("hernia"/exp OR hernia) AND ("pregnancy" / exp OR pregnancy) for EMBASE. We also hand-searched for additional studies through the references of related articles. The search ended in 2020.

\subsection{Study Selection}

We performed a two-step study selection to verify eligibility and inclusion criteria. In the first step, two reviewers (Sang Hun Lee and Jin Young Choi) independently screened the titles and abstracts of all studies for the management of maternal BH during pregnancy. After the screening, the two reviewers then independently assessed the eligibility of fulltext articles. Any discrepancy was resolved through mutual discussion, and a citation was included in our study if both agreed.

We included all primary studies, including case reports and case series, reporting any type of maternal BH (traumatic, congenital, hiatal, or unknown), herniated organ (stomach, small intestine, large intestine, spleen, omentum, pancreas, kidney, or appendix), symptom 
(acute/sub-acute abdominal/pelvic pain, nausea, vomiting, dyspnea, and pain (shoulder, epigastric, chest, or back)), treatment of herniated organs (surgery or conservative treatment), management according to trimester (conservative treatment, operation after delivery, or simultaneous delivery), or adverse maternal and fetal/neonatal outcome. We excluded case reports or case series reporting maternal diaphragmatic hernia in pregnant patients presenting with traumatic, hiatal, and unknown types.

The evidence was summarized and classified using a standard sorting method in Tables S1 and S2. We did not conduct meta-analyses due to the rarity of cases, but the analyses of case reports were summarized narratively regardless of the presence of control groups.

\section{Results}

From January 1941 to December 2020, the search retrieved 3450 papers, 94 of which were deemed eligible, and 42 articles were reviewed. Therefore, we studied 43 cases based on 42 articles that satisfied the inclusion criteria.

Figure 1 presents a PRISMA (Preferred Reporting Items for Systematic Reviews and Meta-Analyses) flowchart with a summary of the search results. Tables 1 and 2 summarizes the clinical features, treatment modalities, and adverse maternal and fetal/neonatal outcomes of all published cases related to maternal $\mathrm{BH}$ during pregnancy. The clinical feature of maternal BH is shown in Table 3. Of the 43 cases, the mean age of presentation was 28.5 years, and the parity was 19 primigravida (43\%), 16 multiparous (36\%), and nine unknown $(21 \%)$. When the clinical picture accompanying the symptoms was diagnosed, the gestational ages of the diaphragmatic hernias were 28 in the antenatal period-one in the first trimester, 13 in the second trimester, and 14 in the third trimester $(65 \%)$, as well as 15 in the postpartum period (35\%). However, four patients were asymptomatic before pregnancy.

Surgical hernia repair was attempted in $58 \%$ of antepartum and $35 \%$ of postpartum cases. In $7 \%$, no surgery was performed. Of those who underwent surgery $(93 \%)$, repair was achieved through a laparotomy $(44 \%)$, thoracotomy $(23 \%)$, combined laparotomy and thoracotomy $(12 \%)$, laparoscopy $(12 \%)$, and assisted thoracoscopy $(2 \%)$.

Maternal BH demonstrated maternal death in $5 \%$ of cases (2/43) and fetal/neonatal loss in 16\% (7/43-five stillborns and two neonatal deaths). The preterm birth rate was $35 \%(15 / 43)$ for maternal $\mathrm{BH}$, resulting from maternal deaths in $13 \%(2 / 15)$ of cases and fetal/neonatal loss in 40\% (6/15) of cases. 44\% (19/43) of cases demonstrated signs of bowel obstruction, ischemia, or perforation of strangulated viscera in the operative field, resulting in maternal deaths in $11 \%(2 / 19)$ of cases and fetal/neonatal loss in $21 \%(4 / 19)$ of cases.

Table 4 summarizes the relation between treatment for maternal BH during pregnancy and the time interval from hernia diagnosis to hernia surgery according to trimesters and adverse maternal and fetal/neonatal outcomes. Our data showed $79 \%(11 / 14)$ in 1 st and 2nd vs. $36 \%(5 / 14)$ in the 3rd trimester for delayed delivery after surgical repair with diagnosing a hernia and $14 \%(2 / 14)$ in 1 st and 2 nd vs. $50 \%(7 / 14)$ in 3rd trimester for simultaneously delivery with surgical repair. Of 11 cases in the 1st and 2nd trimester with delayed delivery after surgical repair with diagnosing hernia, 11 immediately surgery were undergone. Of 8 cases in the 3rd trimester with delayed delivery after surgical repair with diagnosing hernia, 5 immediate surgery and 3 surgical repair with expectant management were undergone. 


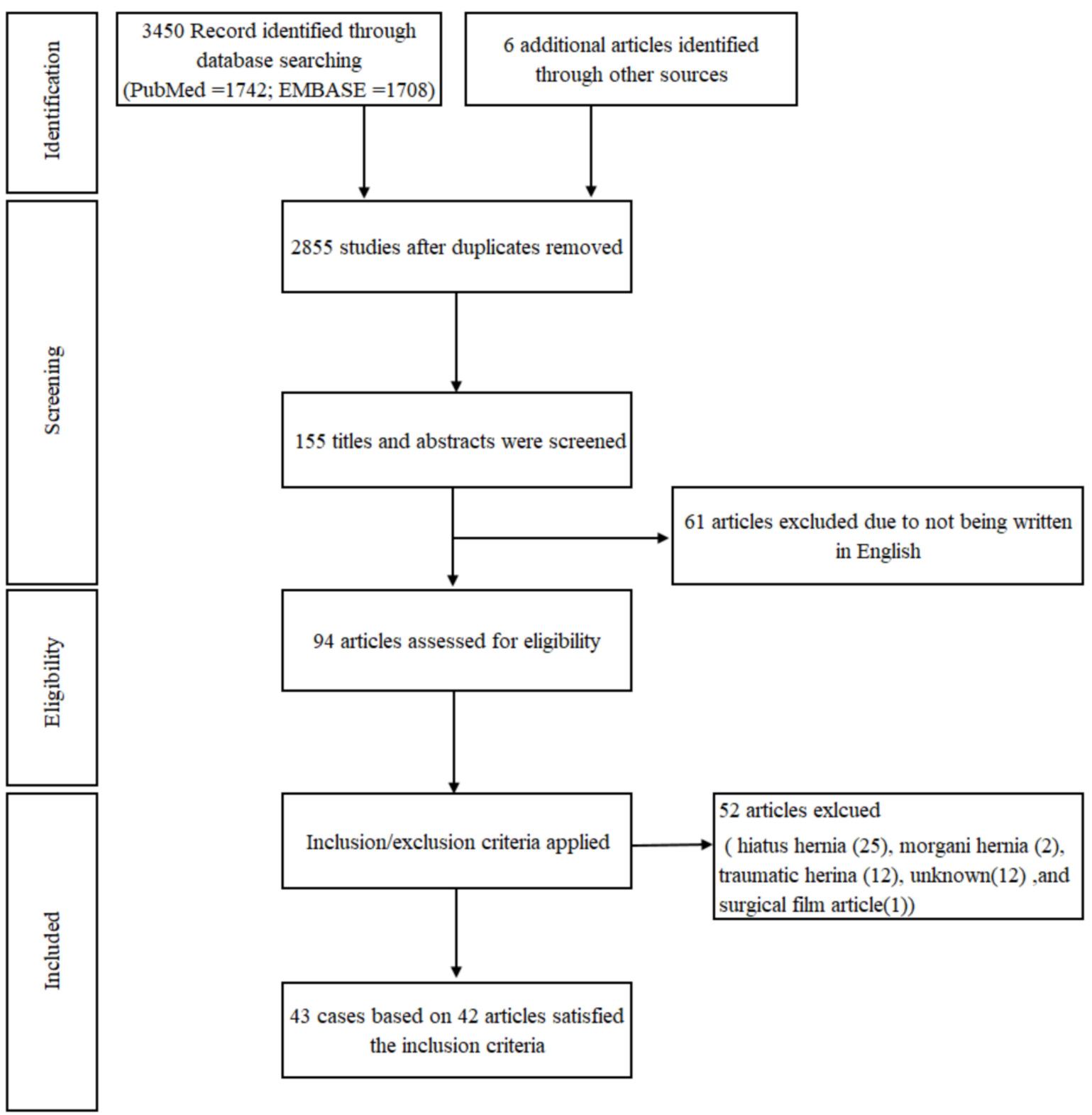

Figure 1. PRISMA (Preferred Reporting Items for Systematic Reviews and Meta-Analyses) flowchart with a summary of the search results. 
Table 1. Literature review from 43 studies investigating maternal Bochdalek hernias (BH) during pregnancy.

\begin{tabular}{|c|c|c|c|c|c|c|c|c|c|c|c|c|c|}
\hline & $\begin{array}{c}\text { Age } \\
\text { (Years) }\end{array}$ & Parity & $\begin{array}{c}\text { Location } \\
\text { of } B H\end{array}$ & Risk Factors & $\begin{array}{c}\text { Surgical } \\
\text { Approach }\end{array}$ & $\begin{array}{c}\text { Gestation at } \\
\text { BH } \\
\text { Diagnosis }\end{array}$ & $\begin{array}{c}\text { Gestation at } \\
\text { Hernia } \\
\text { Repair }\end{array}$ & $\begin{array}{c}\text { The Time } \\
\text { Interval from } \\
\text { Hernia } \\
\text { Diagnosis to } \\
\text { Hernia } \\
\text { Surgery }\end{array}$ & $\begin{array}{c}\text { Gestation } \\
\text { Age at } \\
\text { Delivery }\end{array}$ & $\begin{array}{l}\text { Delivery } \\
\text { Type }\end{array}$ & $\begin{array}{c}\text { Treatment for } \\
\text { Maternal BH } \\
\text { during } \\
\text { Pregnancy }\end{array}$ & $\begin{array}{l}\text { Maternal } \\
\text { Survival }\end{array}$ & $\begin{array}{l}\text { Fetal/Neonatal } \\
\text { Survival }\end{array}$ \\
\hline Thompson, 1945 [4] & 31 & G2P1 & Left & None & Laparotomy & Postpartum & Postpartum & $\begin{array}{l}\text { Immediate } \\
\text { repair }\end{array}$ & NA & VD & $\begin{array}{c}\text { Surgical } \\
\text { repair } \\
\text { after delivery }\end{array}$ & Yes & Yes \\
\hline MURLESS, 1947 [6] & 25 & G?P1 & Right & None & No surgery & 3rd trimester & No surgery & No surgery & 3rd trimester & VD & $\begin{array}{l}\text { Conservative } \\
\text { treatment } \\
\text { after delivery }\end{array}$ & Yes & Yes \\
\hline HODGE, 1950 [7] & 36 & NA & Left & None & Laparotomy & 5th month & 5th month & $\begin{array}{c}\text { Immediate } \\
\text { repair }\end{array}$ & $\begin{array}{l}5 \text { months } \\
\text { and } 3 \text { days }\end{array}$ & NA & $\begin{array}{l}\text { Delayed } \\
\text { delivery after } \\
\text { surgical } \\
\text { repair }\end{array}$ & Death * & Stillborn \\
\hline Pearson, 1950 [5] & 31 & G1P0 & Left & None & Laparotomy & Postpartum & Postpartum & $\begin{array}{l}\text { Immediate } \\
\text { repair }\end{array}$ & NA & VD & $\begin{array}{c}\text { Surgical } \\
\text { repair } \\
\text { after delivery }\end{array}$ & Yes & Yes \\
\hline Kushlan, 1951 [8] & 25 & G3P3 & Left & None & No surgery & Postpartum & No surgery & No surgery & NA & VD & $\begin{array}{l}\text { Non-surgical } \\
\text { treatment } \\
\text { after delivery }\end{array}$ & Yes & Yes \\
\hline Osborne, 1953 [9] & 25 & G5P4 & Left & None & No surgery & 17.6 weeks & No surgery & No surgery & 27.4 weeks & NA & $\begin{array}{c}\text { Death among } \\
\text { conservative } \\
\text { treatment } \\
\text { during } \\
\text { antepartum } \\
\text { period }\end{array}$ & Death $^{+}$ & Stillborn \\
\hline Hobbins, 1953 [10] & 18 & G1P0 & Left & None & Thoracotomy & 1st trimester & 2nd trimester & $\begin{array}{l}\text { Immediate } \\
\text { repair }\end{array}$ & 3rd trimester & VD & $\begin{array}{l}\text { Delayed } \\
\text { delivery after } \\
\text { surgical } \\
\text { repair }\end{array}$ & Yes & Yes \\
\hline Flood,1963 [11] & 22 & NA & Left & None & Laparotomy & 7th month & 7th month & $\begin{array}{l}\text { Immediate } \\
\text { repair }\end{array}$ & $\begin{array}{l}7 \text { months } \\
\text { and } 1 \text { day }\end{array}$ & VD & $\begin{array}{l}\text { Delayed } \\
\text { delivery after } \\
\text { surgical } \\
\text { repair }\end{array}$ & Yes & Stillborn \\
\hline Savage, 1968 [12] & 25 & G?P2 & Left & None & Thoracoscopy & Postpartum & Postpartum & $\begin{array}{l}\text { Immediate } \\
\text { repair }\end{array}$ & NA & VD & $\begin{array}{c}\text { Surgical } \\
\text { repair } \\
\text { after delivery }\end{array}$ & Yes & Yes \\
\hline Gimovsky, 1983 [13] & 20 & G1P0 & Left & None & Laparotomy & Intrapartum & Postpartum & $\begin{array}{l}\text { Immediate } \\
\text { repair }\end{array}$ & 28 weeks & $\mathrm{C} / \mathrm{S}$ & $\begin{array}{c}\text { Surgical } \\
\text { repair } \\
\text { after delivery }\end{array}$ & Yes & $\begin{array}{l}\text { Neonatal } \\
\text { death } \$\end{array}$ \\
\hline Reed, 1987 [14] & 18 & G1P0 & Left & None & Thoracotomy & Postpartum & Postpartum & $\begin{array}{c}\text { Conservative, } \\
\text { repair } 4 \text { days } \\
\text { later }\end{array}$ & 36.4 weeks & $\mathrm{C} / \mathrm{S}$ & $\begin{array}{c}\text { Surgical } \\
\text { repair } \\
\text { after delivery }\end{array}$ & Yes & Yes \\
\hline
\end{tabular}


Table 1. Cont.

\begin{tabular}{|c|c|c|c|c|c|c|c|c|c|c|c|c|c|}
\hline & $\begin{array}{c}\text { Age } \\
\text { (Years) }\end{array}$ & Parity & $\begin{array}{c}\text { Location } \\
\text { of } B H\end{array}$ & Risk Factors & $\begin{array}{c}\text { Surgical } \\
\text { Approach }\end{array}$ & $\begin{array}{c}\text { Gestation at } \\
\text { BH } \\
\text { Diagnosis }\end{array}$ & $\begin{array}{c}\text { Gestation at } \\
\text { Hernia } \\
\text { Repair }\end{array}$ & $\begin{array}{c}\text { The Time } \\
\text { Interval from } \\
\text { Hernia } \\
\text { Diagnosis to } \\
\text { Hernia } \\
\text { Surgery } \\
\end{array}$ & $\begin{array}{c}\text { Gestation } \\
\text { Age at } \\
\text { Delivery }\end{array}$ & $\begin{array}{c}\text { Delivery } \\
\text { Type }\end{array}$ & $\begin{array}{l}\text { Treatment for } \\
\text { Maternal BH } \\
\text { during } \\
\text { Pregnancy }\end{array}$ & $\begin{array}{l}\text { Maternal } \\
\text { Survival }\end{array}$ & $\begin{array}{l}\text { Fetal/Neonatal } \\
\text { Survival }\end{array}$ \\
\hline Kurzel, 1988 [15] & 27 & G1P0 & Left & None & Laparotomy & 33 weeks & 33 weeks & $\begin{array}{l}\text { Immediate } \\
\text { repair }\end{array}$ & 36 weeks & $\mathrm{C} / \mathrm{S}$ & $\begin{array}{l}\text { Delayed } \\
\text { delivery after } \\
\text { surgical } \\
\text { repair }\end{array}$ & Yes & Yes \\
\hline Toorians, 1992 [16] & 29 & G?P2 & Right & None & Laparotomy & 15 weeks & 15 weeks & $\begin{array}{l}\text { Immediate } \\
\text { repair }\end{array}$ & 3rd trimester & VD & $\begin{array}{l}\text { Delayed } \\
\text { delivery after } \\
\text { surgical } \\
\text { repair }\end{array}$ & Yes & Yes \\
\hline Hill, 1996 [17] & 30 & G7P5 & Left & None & $\begin{array}{c}\text { Combined } \\
\text { (thoraco- } \\
\text { laparotomy) }\end{array}$ & Postpartum & Postpartum & $\begin{array}{l}\text { Immediate } \\
\text { repair }\end{array}$ & NA & VD & $\begin{array}{c}\text { Surgical } \\
\text { repair } \\
\text { after delivery }\end{array}$ & Yes & Yes \\
\hline Ortega, 1998 [18] & 23 & G1P0 & Left & None & Laparotomy & Postpartum & Postpartum & $\begin{array}{l}\text { Immediate } \\
\text { repair }\end{array}$ & NA & VD & $\begin{array}{c}\text { Surgical } \\
\text { repair } \\
\text { after delivery }\end{array}$ & Yes & Yes \\
\hline Seon, 2002 [19] & NA & G1P0 & Left & None & laparotomy & Postpartum & Postpartum & $\begin{array}{l}\text { Immediate } \\
\text { repair }\end{array}$ & 34 weeks & VD & $\begin{array}{c}\text { Surgical } \\
\text { repair } \\
\text { after delivery }\end{array}$ & Yes & Stillborn \\
\hline Genc, 2003 [21] & 30 & G2P1 & Left & None & Laparotomy & 29 weeks & 31 weeks & $\begin{array}{l}\text { Conservative, } \\
\text { repair } 10 \\
\text { days later }\end{array}$ & 39 weeks & $\mathrm{VD} *$ & $\begin{array}{l}\text { Delayed } \\
\text { delivery after } \\
\text { surgical } \\
\text { repair }\end{array}$ & Yes & Yes \\
\hline Luu, 2006 [22] & 34 & G1P0 & Left & None & Thoracotomy & Postpartum & Postpartum & $\begin{array}{l}\text { Immediate } \\
\text { repair }\end{array}$ & 34 weeks & VD & $\begin{array}{c}\text { Surgical } \\
\text { repair } \\
\text { after delivery }\end{array}$ & Yes & Yes \\
\hline Eglinton, 2006 [23] & 30 & G2P1 & Left & None & Laparotomy & 28 weeks & 28 weeks & $\begin{array}{l}\text { Immediate } \\
\text { repair }\end{array}$ & 28 weeks & $\mathrm{C} / \mathrm{S}$ & $\begin{array}{l}\text { Simultaneously } \\
\text { delivery with } \\
\text { surgical } \\
\text { treatment }\end{array}$ & Yes & Yes \\
\hline Eglinton, 2006 [23] & 29 & G3P2 & Left & None & Laparotomy & 27 weeks & 27 weeks & $\begin{array}{l}\text { Immediate } \\
\text { repair }\end{array}$ & 37 weeks & $\mathrm{C} / \mathrm{S}$ & $\begin{array}{c}\text { Delayed } \\
\text { delivery after } \\
\text { surgical } \\
\text { repair } \\
\end{array}$ & Yes & Yes \\
\hline Barbetakis, 2006 [24] & 31 & G1P0 & Left & None & $\begin{array}{l}\text { Combined } \\
\text { (thoraco- } \\
\text { laparotomy) }\end{array}$ & 23 weeks & 23 weeks & $\begin{array}{l}\text { Immediate } \\
\text { repair }\end{array}$ & 39 weeks & $\mathrm{C} / \mathrm{S}$ & $\begin{array}{l}\text { Delayed } \\
\text { delivery after } \\
\text { surgical } \\
\text { repair }\end{array}$ & Yes & Yes \\
\hline
\end{tabular}


Table 1. Cont.

\begin{tabular}{|c|c|c|c|c|c|c|c|c|c|c|c|c|c|}
\hline & $\begin{array}{c}\text { Age } \\
\text { (Years) }\end{array}$ & Parity & $\begin{array}{c}\text { Location } \\
\text { of } B H\end{array}$ & Risk Factors & $\begin{array}{c}\text { Surgical } \\
\text { Approach }\end{array}$ & $\begin{array}{c}\text { Gestation at } \\
\text { BH } \\
\text { Diagnosis }\end{array}$ & $\begin{array}{c}\text { Gestation at } \\
\text { Hernia } \\
\text { Repair }\end{array}$ & $\begin{array}{c}\text { The Time } \\
\text { Interval from } \\
\text { Hernia } \\
\text { Diagnosis to } \\
\text { Hernia } \\
\text { Surgery } \\
\end{array}$ & $\begin{array}{c}\text { Gestation } \\
\text { Age at } \\
\text { Delivery }\end{array}$ & $\begin{array}{l}\text { Delivery } \\
\text { Type }\end{array}$ & $\begin{array}{c}\text { Treatment for } \\
\text { Maternal BH } \\
\text { during } \\
\text { Pregnancy }\end{array}$ & $\begin{array}{l}\text { Maternal } \\
\text { Survival }\end{array}$ & $\begin{array}{l}\text { Fetal/Neonatal } \\
\text { Survival }\end{array}$ \\
\hline Rajasingam, 2007 [25] & 24 & G1P0 & Left & None & $\begin{array}{l}\text { Combined } \\
\text { (thoraco- } \\
\text { laparotomy) }\end{array}$ & 33.6 weeks & 33.6 weeks & $\begin{array}{l}\text { Immediate } \\
\text { repair }\end{array}$ & 33.6 weeks & $\mathrm{C} / \mathrm{S}$ & $\begin{array}{c}\text { Simultaneous } \\
\text { delivery with } \\
\text { surgical } \\
\text { treatment }\end{array}$ & Yes & Yes \\
\hline Pai, 2007 [26] & 26 & G1P0 & Right & None & Thoracoscopy & Postpartum & Postpartum & $\begin{array}{l}\text { Immediate } \\
\text { repair }\end{array}$ & 3rd trimester & $\mathrm{C} / \mathrm{S}$ & $\begin{array}{l}\text { Surgical } \\
\text { repair after } \\
\text { delivery }\end{array}$ & Yes & Yes \\
\hline Palanivalu, 2008 [27] & 23 & NA & Left & None & Laparoscopy & 6th month & 6th month & $\begin{array}{l}\text { Immediate } \\
\text { repair }\end{array}$ & 9 months & NA & $\begin{array}{l}\text { Delayed } \\
\text { delivery after } \\
\text { surgical } \\
\text { repair }\end{array}$ & Yes & Yes \\
\hline Sano, 2008 [28] & 25 & G1P0 & Left & $\begin{array}{l}\text { Previous } \\
\text { surgery } \Pi\end{array}$ & Laparotomy & 28 weeks & 28 weeks & $\begin{array}{l}\text { Immediate } \\
\text { repair }\end{array}$ & 28 weeks & $\mathrm{C} / \mathrm{S}$ & $\begin{array}{c}\text { Simultaneous } \\
\text { delivery with } \\
\text { surgical } \\
\text { treatment }\end{array}$ & Yes & Yes \\
\hline Hunter, 2009 [29] & 31 & G1P0 & Left & None & Laparotomy & 27 weeks & 27 weeks & $\begin{array}{l}\text { Immediate } \\
\text { repair }\end{array}$ & 27 weeks & $\mathrm{C} / \mathrm{S}$ & $\begin{array}{l}\text { Simultaneous } \\
\text { delivery with } \\
\text { surgical } \\
\text { treatment }\end{array}$ & Yes & Stillborn \\
\hline Islah, 2010 [30] & 30 & G2P1 & Left & None & Laparotomy & 30 weeks & 30 weeks & $\begin{array}{l}\text { Immediate } \\
\text { repair }\end{array}$ & NA & $\mathrm{C} / \mathrm{S}$ & $\begin{array}{l}\text { Delayed } \\
\text { delivery after } \\
\text { surgical } \\
\text { repair }\end{array}$ & Yes & Yes \\
\hline Morcillo, 2010 [31] & 35 & G1P0 & Left & $\underset{\omega}{\text { Known }}$ CHD & Thoracotomy & 15 weeks & 15 weeks & $\begin{array}{l}\text { Immediate } \\
\text { repair }\end{array}$ & 38 weeks & $\mathrm{C} / \mathrm{S}$ & $\begin{array}{l}\text { Delayed } \\
\text { delivery after } \\
\text { surgical } \\
\text { repair }\end{array}$ & Yes & Yes \\
\hline Julien, 2011 [32] & NA & G?P2 & Left & None & Thoracoscopy & 26 weeks & 26 weeks & $\begin{array}{l}\text { Immediate } \\
\text { repair }\end{array}$ & 39 weeks & VD & $\begin{array}{l}\text { Delayed } \\
\text { delivery after } \\
\text { surgical } \\
\text { repair }\end{array}$ & Yes & Yes \\
\hline Ngai, 2012 [33] & 31 & G1P0 & Left & $\underset{\boldsymbol{C}}{\text { Known }}$ CHD & Laparoscopy & 29 weeks & Postpartum & $\begin{array}{l}\text { Conservative, } \\
\text { repair } 11 \\
\text { days later }\end{array}$ & 31 weeks & $\mathrm{C} / \mathrm{S}$ & $\begin{array}{c}\text { Surgical } \\
\text { repair } \\
\text { after delivery }\end{array}$ & Yes & Yes \\
\hline Hamaji, 2013 [34] & 39 & G1P0 & Left & None & Thoracotomy & Postpartum & Postpartum & $\begin{array}{l}\text { Immediate } \\
\text { repair }\end{array}$ & NA & VD & $\begin{array}{c}\text { Surgical } \\
\text { repair } \\
\text { after delivery }\end{array}$ & Yes & Yes \\
\hline
\end{tabular}


Table 1. Cont.

\begin{tabular}{|c|c|c|c|c|c|c|c|c|c|c|c|c|c|}
\hline & $\begin{array}{c}\text { Age } \\
\text { (Years) }\end{array}$ & Parity & $\begin{array}{l}\text { Location } \\
\text { of } B H\end{array}$ & Risk Factors & $\begin{array}{c}\text { Surgical } \\
\text { Approach }\end{array}$ & $\begin{array}{c}\text { Gestation at } \\
\text { BH } \\
\text { Diagnosis }\end{array}$ & $\begin{array}{l}\text { Gestation at } \\
\text { Hernia } \\
\text { Repair }\end{array}$ & $\begin{array}{l}\text { The Time } \\
\text { Interval from } \\
\text { Hernia } \\
\text { Diagnosis to } \\
\text { Hernia } \\
\text { Surgery }\end{array}$ & $\begin{array}{l}\text { Gestation } \\
\text { Age at } \\
\text { Delivery }\end{array}$ & $\begin{array}{l}\text { Delivery } \\
\text { Type }\end{array}$ & $\begin{array}{l}\text { Treatment for } \\
\text { Maternal BH } \\
\text { during } \\
\text { Pregnancy }\end{array}$ & $\begin{array}{l}\text { Maternal } \\
\text { Survival }\end{array}$ & $\begin{array}{l}\text { Fetal/Neonatal } \\
\text { Survival }\end{array}$ \\
\hline Wieman, 2013 [35] & 42 & NA & Right & None & Laparoscopy & 27 weeks & 27 weeks & $\begin{array}{l}\text { Immediate } \\
\text { repair }\end{array}$ & NA & NA & $\begin{array}{c}\text { Delayed } \\
\text { delivery after } \\
\text { surgical } \\
\text { treatment }\end{array}$ & Yes & Yes \\
\hline Ali, 2014 [36] & 25 & G1P0 & Right & None & Thoracotomy & 2nd trimester & 3rd trimester & $\begin{array}{l}\text { Immediate } \\
\text { repair }\end{array}$ & NA & NA & $\begin{array}{l}\text { Delayed } \\
\text { delivery after } \\
\text { surgical } \\
\text { repair }\end{array}$ & Yes & Yes \\
\hline Debergh, 2014 [37] & 38 & G2P1 & Right & None & Laparoscopy & 16 weeks & 16 weeks & $\begin{array}{l}\text { Immediate } \\
\text { repair }\end{array}$ & 3rd trimester & NA & $\begin{array}{l}\text { Delayed } \\
\text { delivery after } \\
\text { surgical } \\
\text { repair }\end{array}$ & Yes & Yes \\
\hline Hernandez, 2015 [38] & 32 & G1P0 & Left & None & Laparotomy & 29 weeks & 32 weeks & $\begin{array}{l}\text { Conservative, } \\
\text { repair } 21 \\
\text { days later }\end{array}$ & 32 weeks & $\mathrm{C} / \mathrm{S}$ & $\begin{array}{l}\text { Simultaneous } \\
\text { delivery with } \\
\text { surgical } \\
\text { treatment }\end{array}$ & Yes & Yes \\
\hline Yetkinel, 2017 [39] & 23 & G2P1 & Left & None & Laparotomy & 29.6 weeks & 30 weeks & $\begin{array}{l}\text { Conservative, } \\
\text { repair } 4 \text { days } \\
\text { later }\end{array}$ & 37.5 weeks & $\mathrm{C} / \mathrm{S}$ & $\begin{array}{l}\text { Delayed } \\
\text { delivery after } \\
\text { surgical } \\
\text { repair }\end{array}$ & Yes & Yes \\
\hline Reddy, 2018 [40] & 30 & G2P0 & Left & None & Thoracotomy & 31.3 weeks & 32.3 weeks & $\begin{array}{l}\text { Conservative, } \\
\text { repair } 7 \text { days } \\
\text { later }\end{array}$ & 32.3 weeks & $\mathrm{C} / \mathrm{S}$ & $\begin{array}{l}\text { Simultaneous } \\
\text { delivery with } \\
\text { surgical } \\
\text { treatment }\end{array}$ & Yes & Yes \\
\hline Matsudera, 2018 [41] & 26 & NA & Left & None & laparoscopy & Postpartum & Postpartum & $\begin{array}{l}\text { Immediate } \\
\text { repair }\end{array}$ & NA & NA & $\begin{array}{l}\text { Surgical } \\
\text { repair after } \\
\text { delivery }\end{array}$ & Yes & Yes \\
\hline Vasquez, 2019 [42] & 29 & NA & Left & None & $\begin{array}{l}\text { Combined } \\
\text { (thoraco- } \\
\text { laparotomy) }\end{array}$ & 26.3 weeks & 27.4 weeks & $\begin{array}{c}\text { Conservative, } \\
\text { repair } 8 \text { days } \\
\text { later }\end{array}$ & 27.4 weeks & $\mathrm{C} / \mathrm{S}$ & $\begin{array}{l}\text { Delayed } \\
\text { delivery after } \\
\text { surgical } \\
\text { repair }\end{array}$ & Yes & Yes \\
\hline Suhardja, 2019 [43] & 30 & NA & Left & None & Thoracotomy & 31 weeks & 32 weeks & $\begin{array}{c}\text { Conservative, } \\
\text { repair } 7 \text { days } \\
\text { later }\end{array}$ & 32 weeks & $\mathrm{C} / \mathrm{S}$ & $\begin{array}{l}\text { Simultaneous } \\
\text { delivery with } \\
\text { surgical } \\
\text { treatment }\end{array}$ & Yes & Yes \\
\hline Ménassa, 2019 [44] & 30 & G4P2 & Left & None & Laparotomy & 35 weeks & 35 weeks & $\begin{array}{l}\text { Immediate } \\
\text { repair }\end{array}$ & 35 weeks & $\mathrm{C} / \mathrm{S}$ & $\begin{array}{l}\text { Simultaneous } \\
\text { delivery with } \\
\text { surgical } \\
\text { treatment }\end{array}$ & Yes & Yes \\
\hline
\end{tabular}


Table 1. Cont.

\begin{tabular}{|c|c|c|c|c|c|c|c|c|c|c|c|c|c|}
\hline & $\begin{array}{c}\text { Age } \\
\text { (Years) }\end{array}$ & Parity & $\begin{array}{c}\text { Location } \\
\text { of } \mathrm{BH}\end{array}$ & Risk Factors & $\begin{array}{l}\text { Surgical } \\
\text { Approach }\end{array}$ & $\begin{array}{c}\text { Gestation at } \\
\text { BH } \\
\text { Diagnosis }\end{array}$ & $\begin{array}{c}\text { Gestation at } \\
\text { Hernia } \\
\text { Repair }\end{array}$ & $\begin{array}{c}\text { The Time } \\
\text { Interval from } \\
\text { Hernia } \\
\text { Diagnosis to } \\
\text { Hernia } \\
\text { Surgery }\end{array}$ & $\begin{array}{c}\text { Gestation } \\
\text { Age at } \\
\text { Delivery }\end{array}$ & $\begin{array}{c}\text { Delivery } \\
\text { Type }\end{array}$ & $\begin{array}{c}\text { Treatment for } \\
\text { Maternal BH } \\
\text { during } \\
\text { Pregnancy }\end{array}$ & $\begin{array}{l}\text { Maternal } \\
\text { Survival }\end{array}$ & $\begin{array}{l}\text { Fetal/Neonatal } \\
\text { Survival }\end{array}$ \\
\hline Haj-Yahia, 2020 [45] & 29 & NA & Left & None & Laparotomy & Postpartum & Postpartum & $\begin{array}{l}\text { Immediate } \\
\text { repair }\end{array}$ & 3rd trimester & $\mathrm{C} / \mathrm{S}$ & $\begin{array}{c}\text { Surgical } \\
\text { tratment } \\
\text { after delivery }\end{array}$ & Yes & $\begin{array}{c}\text { Neonatal } \\
\text { death } \|\end{array}$ \\
\hline
\end{tabular}

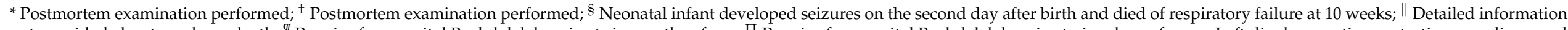

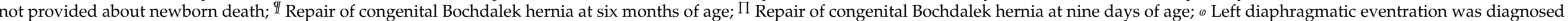

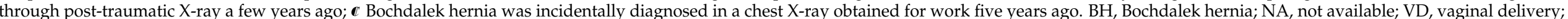

$\mathrm{C} / \mathrm{S}$, cesarean section.

Table 2. Literature review from 43 studies investigating maternal Bochdalek hernia during pregnancy.

\begin{tabular}{|c|c|c|c|c|c|c|c|}
\hline Author and Date & Age (Years) & Presenting Symptoms & $\begin{array}{c}\text { Hernia Defect Size } \\
(\mathrm{cm})\end{array}$ & Herniated Organs & $\begin{array}{c}\text { Bowel Obstruction, Ischemia, } \\
\text { Perforation of Herniated Organs }\end{array}$ & $\begin{array}{l}\text { Maternal } \\
\text { Survival }\end{array}$ & $\begin{array}{c}\text { Fetal/Neonatal } \\
\text { Survival }\end{array}$ \\
\hline Thompson, 1945 [4] & 31 & Epigastric pain, nausea, vomiting, restless & 10 & Stomach, intestine, pancreas & Negative & Yes & Yes \\
\hline Murless, 1947 [6] & 25 & Epigastric pain, shortness of breath & NA & Not stated & NA & Yes & Yes \\
\hline Hodge, 1950 [7] & 36 & Epigastric pain, regurgitation of food, dyspnea & NA & Stomach & Positive & Death & Stillborn \\
\hline Pearson, 1950 [5] & 31 & Abdominal pain, chest pain, nausea, vomiting & 7 & Stomach & Negative & Yes & Yes \\
\hline Kushlan, 1951 [8] & 25 & $\begin{array}{l}\text { Abdominal pain, scapula pain, nausea, } \\
\text { vomiting }\end{array}$ & NA & Not stated & NA & Yes & Yes \\
\hline Osborne, 1953 [9] & 25 & Headache, chills, fever, vomiting & 6 & Stomach, transverse colon & Positive & Death & Stillborn \\
\hline Hobbins, $1953[10]$ & 18 & Abdominal pain, vomiting & 1 & $\begin{array}{l}\text { The colon of the splenic flexure, } \\
\text { transverse colon, mesentery, omentum }\end{array}$ & Negative & Yes & Yes \\
\hline Flood,1963 [11] & 22 & Dyspnea, respiratory distress & NA & Stomach, colon, cecum & Negative & Yes & Stillborn \\
\hline Savage, 1968 [12] & 25 & Epigastric pain, back pain, vomiting, dyspnea & 10 & $\begin{array}{l}\text { Stomach, descending colon, omentum, } \\
\text { splenic flexure }\end{array}$ & Positive & Yes & Yes \\
\hline Gimovsky, 1983 [13] & 20 & $\begin{array}{l}\text { Abdominal pain, chest pain, diarrhea, myalgia, } \\
\text { uterine contraction }\end{array}$ & NA & Transverse colon & Positive & Yes & Neonatal death \\
\hline Reed, 1987 [14] & 18 & Abdominal pain, vomiting & 12 & Stomach & Positive & Yes & Yes \\
\hline Kurzel, 1988 [15] & 27 & Chest pain, epigastric pain, nausea, vomiting & 14 & $\begin{array}{l}\text { Stomach, small intestine, transverse } \\
\text { and ascending colon, pancreas, splenic } \\
\text { flexure, left kidney }\end{array}$ & Negative & Yes & Yes \\
\hline Toorians, 1992 [16] & 29 & Vomiting, chest pain, cough & 7 & Transverse colon, omentum & Positive & Yes & Yes \\
\hline Hill, 1996 [17] & 30 & Back pain, difficulty breathing & 5 & Small intestine, large intestine & Positive & Yes & Yes \\
\hline Ortega, 1998 [18] & 23 & Cardiorespiratory failure & 6 & Small intestine, transverse colon & Negative & Yes & Yes \\
\hline
\end{tabular}


Table 2. Cont

\begin{tabular}{|c|c|c|c|c|c|c|c|}
\hline Author and Date & Age (Years) & Presenting Symptoms & $\begin{array}{l}\text { Hernia Defect Size } \\
(\mathrm{cm})\end{array}$ & Herniated Organs & $\begin{array}{l}\text { Bowel Obstruction, Ischemia, } \\
\text { Perforation of Herniated Organs }\end{array}$ & $\begin{array}{l}\text { Maternal } \\
\text { Survival }\end{array}$ & $\begin{array}{l}\text { Fetal/Neonatal } \\
\text { Survival }\end{array}$ \\
\hline Seon, $2002[19]$ & NA & $\begin{array}{l}\text { Nausea, vomiting, shoulder tip, left chest, and } \\
\text { hypochondrium pain }\end{array}$ & 2 & Stomach & Negative & Yes & Stillborn \\
\hline Williams, 2003 [20] & 32 & Vomiting, abdominal pain & NA & Stomach, omentum & Positive & Yes & Yes \\
\hline Genc, 2003 [21] & 30 & Nausea, vomiting, epigastric pain & 8 & Stomach, transverse colon & Negative & Yes & Yes \\
\hline Luu, 2006 [22] & 34 & Nausea, vomiting, back pain & 2 & Stomach & Positive & Yes & Yes \\
\hline Eglinton, 2006 [23] & 30 & Epigastric pain, chest pain & NA & Stomach & Positive & Yes & Yes \\
\hline Eglinton, 2006 [23] & 29 & Epigastric pain, vomiting & NA & $\begin{array}{l}\text { Stomach, transverse colon, splenic } \\
\text { flexure }\end{array}$ & Positive & Yes & Yes \\
\hline Barbetakis, 2006 [24] & 31 & $\begin{array}{l}\text { Dyspnea, nausea, vomiting, epigastric pain, } \\
\text { weight loss }\end{array}$ & 7 & $\begin{array}{l}\text { Stomach, transverse and ascending } \\
\text { colon, omentum }\end{array}$ & Positive & Yes & Yes \\
\hline Rajasingam, 2007 [25] & 24 & Vomiting, shoulder tip, and abdominal pain & NA & Stomach & Positive & Yes & Yes \\
\hline Pai, 2007 [26] & 26 & Breathlessness, chest pain & NA & $\begin{array}{l}\text { Liver hepatic flexure, ascending and } \\
\text { transverse colon }\end{array}$ & Negative & Yes & Yes \\
\hline Palanivalu, 2008 [27] & 23 & Nausea, oliguria, breathlessness & 5 & Stomach, large intestine & Negative & Yes & Yes \\
\hline Sano, 2008 [28] & 25 & Tachypnea, dyspnea, chest pain & 2 & Small intestine, omentum & Positive & Yes & Yes \\
\hline Hunter, 2009 [29] & 31 & Abdominal pain & NA & $\begin{array}{l}\text { Stomach, small intestine, transverse } \\
\text { colon, splenic flexure }\end{array}$ & Negative & Yes & Stillborn \\
\hline Morcillo, 2010 [31] & 35 & Chest pain, dyspnea & 15 & Stomach, omentum, large intestine & Negative & Yes & Yes \\
\hline Julien, 2011 [32] & NA & Nausea, vomiting, epigastric pain & NA & Large intestine, small intestine & Negative & Yes & Yes \\
\hline Ngai, 2012 [33] & 31 & $\begin{array}{c}\text { Abdominal pain, shortness of breath, nausea, } \\
\text { vomiting }\end{array}$ & NA & $\begin{array}{c}\text { Stomach, small intestine, large } \\
\text { intestine, splenic flexure, pancreatic } \\
\text { body }\end{array}$ & Negative & Yes & Yes \\
\hline Hamaji, 2013 [34] & 39 & Epigastric pain, nausea, vomiting & 5 & Transverse colon, omentum & Negative & Yes & Yes \\
\hline Wieman, 2013 [35] & 42 & Chest pain, shoulder pain, abdominal pain & 7 & Large intestine, cecum & Positive & Yes & Yes \\
\hline Ali, 2014 [36] & 25 & Dyspnea & NA & Small intestine & Negative & Yes & Yes \\
\hline Debergh, 2014 [37] & 38 & Abdominal pain, nausea, vomiting & 5 & Small intestine & Positive & Yes & Yes \\
\hline Hernandez, 2015 [38] & 32 & Nausea, vomiting, epigastric pain & 6 & $\begin{array}{l}\text { Ascending and transverse colon, small } \\
\text { intestine }\end{array}$ & Negative & Yes & Yes \\
\hline Yetkinel, 2017 [39] & 23 & Abdominal pain & 4 & Transverse colon & Negative & Yes & Yes \\
\hline Reddy, 2018 [40] & 30 & Epigastric pain, chest pain, nausea, vomiting & NA & $\begin{array}{c}\text { Stomach, small intestine, large } \\
\text { intestine, appendix, splenic flexure, } \\
\text { omentum }\end{array}$ & Negative & Yes & Yes \\
\hline Matsudera, 2018 [41] & 26 & Abdominal pain, dyspnea & 7 & $\begin{array}{l}\text { Stomach, splenic flexure, small } \\
\text { intestine, descending colon }\end{array}$ & Negative & Yes & Yes \\
\hline
\end{tabular}


Table 2. Cont.

\begin{tabular}{|c|c|c|c|c|c|c|c|}
\hline Author and Date & Age (Years) & Presenting Symptoms & $\begin{array}{c}\text { Hernia Defect Size } \\
(\mathrm{cm})\end{array}$ & Herniated Organs & $\begin{array}{l}\text { Bowel Obstruction, Ischemia, } \\
\text { Perforation of Herniated Organs }\end{array}$ & $\begin{array}{l}\text { Maternal } \\
\text { Survival }\end{array}$ & $\begin{array}{l}\text { Fetal/Neonatal } \\
\text { Survival }\end{array}$ \\
\hline Vasquez, 2019 [42] & 29 & Epigastric pain, vomiting & NA & Large intestine, stomach & Positive & Yes & Yes \\
\hline Suhardja, 2019 [43] & 30 & Abdominal pain, nausea, vomiting, cough & NA & $\begin{array}{l}\text { Stomach, small intestine, cecum, } \\
\text { splenic flexure, appendix, transverse } \\
\text { colon }\end{array}$ & Negative & Yes & Yes \\
\hline Ménassa, 2019 [44] & 30 & Epigastric pain, anorexia, nausea, vomiting & NA & $\begin{array}{l}\text { Stomach, small intestine, transverse } \\
\text { colon }\end{array}$ & Positive & Yes & Yes \\
\hline Haj-Yahia, 2020 [45] & 29 & Sudden cardiac arrest during cesarean section & NA & $\begin{array}{c}\text { Stomach, transverse colon, pancreas, } \\
\text { omentum }\end{array}$ & Negative & Yes & Neonatal death \\
\hline
\end{tabular}


Table 3. Clinical features of the 43 reviewed cases of maternal BH complicating pregnancy.

\begin{tabular}{|c|c|}
\hline Mean Age (Years) & 28.5 \\
\hline \multicolumn{2}{|l|}{ Gestational age when maternal $\mathrm{BH}$ was diagnosed by imaging } \\
\hline First trimester & $1(2 \%)$ \\
\hline Second trimester & $13(30 \%)$ \\
\hline Third trimester & $14(33 \%)$ \\
\hline Postpartum & $15(35 \%)$ \\
\hline \multicolumn{2}{|l|}{ Parity } \\
\hline Primigravida & $19(44 \%)$ \\
\hline Multiparous & $16(37 \%)$ \\
\hline NA & $8(19 \%)$ \\
\hline \multicolumn{2}{|l|}{ Location of maternal BH } \\
\hline Right side & $6(14 \%)$ \\
\hline Left side & $37(86 \%)$ \\
\hline Hernia defect size $(\mathrm{cm})$ & 5.6 \\
\hline \multicolumn{2}{|l|}{ Number of herniated organs } \\
\hline 1 organ & $9(21 \%)$ \\
\hline 2 or 3 organs & $18(42 \%)$ \\
\hline$>3$ organs & $14(33 \%)$ \\
\hline NA & $2(46 \%)$ \\
\hline \multicolumn{2}{|l|}{ Type of herniated organs } \\
\hline Stomach & $27(63 \%)$ \\
\hline Small bowel & $14(33 \%)$ \\
\hline Colon & $30(70 \%)$ \\
\hline Spleen & $9(21 \%)$ \\
\hline Pancreas & $4(9 \%)$ \\
\hline Omentum & $11(26 \%)$ \\
\hline Cecum & $3(7 \%)$ \\
\hline kidney & $1(2 \%)$ \\
\hline Liver & $1(2 \%)$ \\
\hline Appendix & $3(7 \%)$ \\
\hline \multicolumn{2}{|l|}{ Surgical methods } \\
\hline Laparotomy & $19(44 \%)$ \\
\hline Thoracotomy & $10(23 \%)$ \\
\hline Laparoscopy & $5(12 \%)$ \\
\hline Assisted thoracoscopy & $1(2 \%)$ \\
\hline Laparotomy and thoracotomy & $5(12 \%)$ \\
\hline Non-surgical treatment & $3(7 \%)$ \\
\hline \multicolumn{2}{|l|}{ Gestational age at hernia surgery } \\
\hline Antepartum period & $25(58 \%)$ \\
\hline Postpartum period & $15(35 \%)$ \\
\hline Non-surgical treatment & $3(7 \%)$ \\
\hline \multicolumn{2}{|l|}{ Hernia repair methods } \\
\hline Simple suture & $28(65 \%)$ \\
\hline Suture with mesh & $9(21 \%)$ \\
\hline Mesh only & $1(2 \%)$ \\
\hline No repair & $3(7 \%)$ \\
\hline Unknown & $2(5 \%)$ \\
\hline \multicolumn{2}{|l|}{ Mortality } \\
\hline Fetal/neonatal death & $7(16 \%)$ \\
\hline Maternal death & $2(5 \%)$ \\
\hline \multicolumn{2}{|l|}{ Mode of delivery type } \\
\hline Normal delivery & $16(37 \%)$ \\
\hline Cesarean delivery & $20(47 \%)$ \\
\hline Unknown & $7(16 \%)$ \\
\hline Bowel obstruction, ischemia, perforation of herniated organs & $19(44 \%)$ \\
\hline \multicolumn{2}{|l|}{ Pregnancy Outcomes } \\
\hline Preterm birth & $15(35 \%)$ \\
\hline Full term delivery & $16(37 \%)$ \\
\hline Unknown & $12(28 \%)$ \\
\hline
\end{tabular}

$\overline{\mathrm{BH}}$, Bochdalek hernia; NA, not available; VD, vaginal delivery; C/S, cesarean section. 
Table 4. Treatment of herniated organs, management according to trimester, and adverse maternal and fetal/neonatal outcomes of maternal BH during pregnancy.

\begin{tabular}{|c|c|c|c|c|c|c|c|c|c|}
\hline & \multirow{2}{*}{\multicolumn{2}{|c|}{$\begin{array}{c}\text { Treatment for Maternal BH } \\
\text { during Pregnancy }\end{array}$}} & \multirow{2}{*}{\multicolumn{2}{|c|}{$\begin{array}{c}\text { The Time Interval from Hernia } \\
\text { Diagnosis to Hernia Surgery }\end{array}$}} & \multicolumn{3}{|c|}{$\begin{array}{l}\text { Bowel Obstruction, Ischemia, } \\
\text { Perforation of Herniated Organs }\end{array}$} & \multicolumn{2}{|c|}{ Mortality } \\
\hline & & & & & Positive & Negative & Unknown & $\begin{array}{l}\text { Maternal } \\
\text { Death }\end{array}$ & $\begin{array}{c}\text { Fetal/ } \\
\text { Neonatal } \\
\text { Death }\end{array}$ \\
\hline \multirow{5}{*}{$\begin{array}{l}\text { 1st and 2nd } \\
\text { trimester }(n=14)\end{array}$} & \multirow[b]{2}{*}{$\begin{array}{l}\text { Delayed delivery } \\
\text { after hernia surgery }\end{array}$} & \multirow[b]{2}{*}{11} & $\begin{array}{l}\text { Immediate hernia } \\
\text { surgery after } \\
\text { diagnosis }\end{array}$ & 11 & $6^{a}$ & $5^{b}$ & 0 & 1 & 1 \\
\hline & & & $\begin{array}{l}\text { Delayed hernia } \\
\text { surgery after } \\
\text { expectant } \\
\text { management }\end{array}$ & 0 & 0 & 0 & 0 & 0 & 0 \\
\hline & \multirow{2}{*}{$\begin{array}{l}\text { Simultaneous } \\
\text { delivery with } \\
\text { hernia surgery }\end{array}$} & \multirow[b]{2}{*}{2} & $\begin{array}{l}\text { Immediate hernia } \\
\text { surgery after } \\
\text { diagnosis }\end{array}$ & 1 & 0 & $1^{c}$ & 0 & 0 & 1 \\
\hline & & & $\begin{array}{l}\text { Delayed hernia } \\
\text { surgery after } \\
\text { expectant } \\
\text { management }\end{array}$ & 1 & $1^{\mathrm{d}}$ & 0 & 0 & 0 & 0 \\
\hline & $\begin{array}{l}\text { Non-surgical } \\
\text { treatment after } \\
\text { delivery }\end{array}$ & 1 & & & $1^{\mathrm{e}}$ & 0 & 0 & 1 & 1 \\
\hline \multirow{7}{*}{$\begin{array}{l}\text { 3rd trimester } \\
\quad(n=14)\end{array}$} & \multirow[b]{2}{*}{$\begin{array}{l}\text { Delayed delivery } \\
\text { after hernia surgery }\end{array}$} & \multirow[b]{2}{*}{5} & $\begin{array}{l}\text { Immediate hernia } \\
\text { surgery after } \\
\text { diagnosis }\end{array}$ & 3 & $1^{\mathrm{f}}$ & $2^{g}$ & 0 & 0 & 1 \\
\hline & & & $\begin{array}{l}\text { Delayed hernia } \\
\text { surgery after } \\
\text { expectant } \\
\text { management }\end{array}$ & 2 & 0 & $2^{h}$ & 0 & 0 & 0 \\
\hline & \multirow{2}{*}{$\begin{array}{l}\text { Simultaneous } \\
\text { delivery with } \\
\text { hernia surgery }\end{array}$} & \multirow[b]{2}{*}{7} & $\begin{array}{l}\text { Immediate hernia } \\
\text { surgery after } \\
\text { diagnosis }\end{array}$ & 4 & $4^{\mathrm{i}}$ & 0 & 0 & 0 & 0 \\
\hline & & & $\begin{array}{l}\text { Delayed hernia } \\
\text { surgery after } \\
\text { expectant } \\
\text { management }\end{array}$ & 3 & 0 & $3^{j}$ & 0 & 0 & 0 \\
\hline & \multirow{2}{*}{$\begin{array}{c}\text { Hernia surgery } \\
\text { after delivery }\end{array}$} & \multirow[b]{2}{*}{1} & $\begin{array}{l}\text { Immediate hernia } \\
\text { surgery after } \\
\text { diagnosis }\end{array}$ & 0 & 0 & 0 & 0 & 0 & 0 \\
\hline & & & $\begin{array}{l}\text { Delayed hernia } \\
\text { surgery after } \\
\text { expectant } \\
\text { management }\end{array}$ & 1 & 0 & $1^{\mathrm{k}}$ & 0 & 0 & 0 \\
\hline & $\begin{array}{l}\text { Non-surgical } \\
\text { treatment after } \\
\text { delivery }\end{array}$ & 1 & & & 0 & 0 & 1 & 0 & 0 \\
\hline \multirow{5}{*}{$\begin{array}{l}\text { Postpartum * } \\
\quad(n=15)\end{array}$} & \multirow[b]{2}{*}{$\begin{array}{l}\text { Hernia surgery } \\
\text { after delivery }\end{array}$} & \multirow[b]{2}{*}{13} & $\begin{array}{l}\text { Immediate hernia } \\
\text { surgery after } \\
\text { diagnosis }\end{array}$ & 11 & $4^{1}$ & $7^{\mathrm{m}}$ & 0 & 0 & 2 \\
\hline & & & $\begin{array}{l}\text { Delayed hernia } \\
\text { surgery after } \\
\text { expectant } \\
\text { management }\end{array}$ & 2 & $1^{\mathrm{n}}$ & $1^{\circ}$ & 0 & 0 & 0 \\
\hline & \multirow{2}{*}{$\begin{array}{l}\text { Simultaneous } \\
\text { delivery with } \\
\text { hernia surgery }\end{array}$} & \multirow[b]{2}{*}{1} & $\begin{array}{l}\text { Immediate hernia } \\
\text { surgery after } \\
\text { diagnosis }\end{array}$ & 1 & $1^{\mathrm{p}}$ & 0 & 0 & 0 & 1 \\
\hline & & & $\begin{array}{l}\text { Delayed hernia } \\
\text { surgery after } \\
\text { expectant } \\
\text { management }\end{array}$ & 0 & 0 & 0 & 0 & 0 & 0 \\
\hline & $\begin{array}{l}\text { Non-surgical } \\
\text { treatment after } \\
\text { delivery }\end{array}$ & 1 & & 1 & 0 & 0 & 1 & 0 & 0 \\
\hline
\end{tabular}

First trimester defined as 0-13 6/7 weeks of gestation from last menstrual period; second trimester defined as 14 0/7-27 6/7 weeks of gestation from last menstrual period; third trimester defined as $280 / 7-420 / 7$ weeks of gestation from last menstrual period. Wks, weeks; $\mathrm{h}$, hours. * One intrapartum case. BH, Bochdalek hernia; NA, not available; VD, vaginal delivery; C/S, cesarean section. ${ }^{\mathrm{a}}[7,16,23,24,35,37]$; b $[10,27,31,32,36] ;{ }^{\mathrm{c}}[29] ;{ }^{\mathrm{d}}[42] ;{ }^{\mathrm{e}}[9] ;{ }^{\mathrm{f}}[30] ;{ }^{\mathrm{g}}[11,15]{ }^{\mathrm{h}}[21,39] ;{ }^{\mathrm{i}}[23,25,28,44] ;{ }^{\mathrm{j}}[11,15,30] ;{ }^{\mathrm{k}}[33]{ }^{\mathrm{l}}{ }^{\mathrm{l}}[4,12,17,19,22] ;{ }^{\mathrm{m}}[4,5,18,26,34,41,45] ;{ }^{\mathrm{n}}[14]$; ${ }^{o}[20] ; \mathrm{p}[13]$. 


\section{Discussion}

\subsection{Embryology and Pathogenesis}

In the 18th and 19th centuries, the renowned anatomists and pathologists Giovanni Battista Morgagni and Alexander Bochdalek dedicated themselves to elucidating the development of anatomy. The anteromedial defect of diaphragmatic hernia was named Morgani in 1765, while a congenital postero-lateral diaphragmatic defect was named Bochdalek in 1848.

The diaphragm is a thin skeletal muscle located at the base of the thorax between the abdomen and the thorax, consisting of a thin central aponeurosis and a peripheral muscle. The muscular diaphragm begins developing at the 3rd week of embryologic development and is fully formed at the 12th week.

The normal diaphragm development is formed by organogenesis from (1) the transverse septum, formed during the third embryonic week that eventually forms the central tendon, (2) the right and left pleuroperitoneal membranes closed with the dorsal mesentery of the esophagus medially, the transverse septum laterally and caudally, and body wall posteriorly at approximately the 6th week of the embryonic period. (3) the dorsal mesentery of the esophagus forming the crura of the diaphragm, (4) inter and outer body wall muscles splitting the pleural cavities and their costodiaphragmatic fold during the 9th and 12th embryonic weeks.

$\mathrm{CDH}$ account for most of the developmental abnormality of the diaphragm. Developmental defects in the fetus's diaphragm in utero may range from weak-to-partial to complete.

The pathogenesis of $\mathrm{CDH}$ still is not well known and usually exhibits sporadic patterns; however, teratogenic and genetic factors can affect it. According to embryonic theory, a CDH may result from a delay or variation in the organogenesis timetable between diaphragmatic components and abdominal viscera.

By the end of the 6th week of the embryonic period, the primordial diaphragm originated from four diaphragmatic components is produced. Failure of fusion of composite structure related to the primordial diaphragm is the most common causal factor of $\mathrm{CDH}$. $\mathrm{CDH}$ is characterized by the presence of abdominal viscera in the thoracic cavity. During the 10th week, the intestines return to the abdomen. If some component of the primordial diaphragm is still open and has the failure of closure when the intestine returns the abdomen from the umbilical cord at this period. The herniation arises as to the protrusion of the abdominal viscera into the thorax.

The $\mathrm{CDH}$ has a prevalence of $0.8-5 / 10,000$ in live births. The incidence in the prenatal period may be much higher due to the effects of fetuses that die in utero without a pathological postmortem. This occurs equally for both males and females. The Bochdalek hernia(postero-lateral hernia) represents $70-75 \%$ of all $\mathrm{CDH}$, while the Morgagni hernia (anterior hernia) 23-28\%, central hernias 2-7\%, and the other types (congenital epigastric hernia, hiatal hernia, and eventration of the diaphragm) [46-48].

A Bochdalek hernia is caused by the defective development of the pleuroperitoneal membrane in the posterolateral portion of the diaphragm. The location is $85 \%$ on the left and $13 \%$ on the right, with $2 \%$ of cases presenting bilaterally [49,50]. In an adult, the defect is $78 \%$ on the left, $20 \%$ on the right, and $2 \%$ bilateral [51]. Our data also showed left-side dominance for infants and adults.

The pleuroperitoneal fold (PPF) is two pyramidal-shaped mesodermal structures between the abdominal and thoracic cavities and forms the dorsal-lateral portions of the primordial diaphragm [52]. Recently, more studies have suggested that diaphragmatic defects occur earlier in the developmental period of PPF than in the period associated with fusion. A "mesenchymal hit" hypothesis has been suggested to explain the effects in the PPF of muscle connective tissue fibroblasts and somatic genetic mutations associated with $\mathrm{CDH}$. In experimental animal models, an impaired PPF of cellular etiology and genetic mutations of transcription factors such as GATA-4 and its coregulator FOG-2 appear to be potent causes for abnormality and CDHs [52,53]. 
Thus far, the pathogenesis of $\mathrm{CDHs}$ is thought to be complex, involving multiple genetic factors (e.g., Wilms tumor gene (wt1) and COUP-TFII) [54-56], environmental toxins (e.g., herbicides and nitrogen) [57], and nutritional deficiencies (e.g., vitamin A) [58]

\subsection{Etiology}

Carl Hedblom [2] systematically classified diaphragmatic hernias into the following four etiologies: congenital, caused by an imperfect developmental defect of the diaphragm; traumatic, the result of various causes (e.g., knife wound, crushing injury, fall, collision, or fractured rib); acquired, caused by gradual irritation of the anatomical region with the least resistance such as the esophageal ring; and indeterminate. A hernia may be classified into the congenital type due to developmental disabilities in the embryonic period, acquired type due to gradual irritational pressure or sudden trauma, and mixed type.

A Bochdalek hernia in an adult can stem from increased intra-abdominal pressure, such as pregnancy, chronic constipation, severe coughing, binge eating, fits of laughter, upside-down hanging, or diving.

In 173 reviewed cases, Brown et al. [51] reported that precipitating factors or triggering events accounted for $25 \%$ of $\mathrm{BH}$ cases and that pregnancy was the most common cause, accounting for $34 \%$ of cases with one or more triggering factors or $8 \%$ overall. Our data showed that pregnancy-associated maternal BHs occur regardless of maternal age or parity (primigravida $43 \%$, multiparous $36 \%$, and unknown $21 \%$ ).

Although labor pain is the major precipitating factor manifesting symptoms, 28/43 cases $(65 \%)$ presented in the antenatal period (one in the first trimester, 13 in the second trimester, and 14 in the third trimester) and postpartum $(15 ; 35 \%)$. Symptoms occurred more frequently in the antenatal period. In this review, the second trimester $(30 \%)$ had as significant a proportion as the third trimester or postpartum. One study reported a rare case for the first trimester [10].

However, four cases were primigravida with no symptoms before pregnancies: Two cases of maternal BH surgically repair in the neonate period [20,28], and two cases of maternal $\mathrm{BH}$ incidentally recognized through chest $\mathrm{X}$-rays five years and several years earlier, respectively $[31,33]$.

\subsection{Clinical Presentation}

The clinical symptoms of patients with diaphragmatic hernias depend primarily on the degree of protrusion of the herniated organs into the chest. Therefore, most symptoms are related to the abdomen and chest and range from no symptoms to life-threatening complication.

Abdominal symptoms include nausea, vomiting, abdominal pain, epigastric pain, and stress after eating. Thoracic symptoms include dyspnea, dysphagia, and pain in the left shoulder, chest, and back. Some authors have reported that vomiting $(60 \%)$, abdominal pain $(57 \%)$, and dyspnea $(57 \%)$ were the most prominent in maternal diaphragmatic hernia during pregnancy. This review demonstrated vomiting (56\%), nausea $(44 \%)$, dyspnea (33\%), epigastric pain $(37 \%)$, abdominal pain $(33 \%)$, chest pain $(23 \%)$, shoulder pain $(9 \%)$, and back pain $(7 \%)$.

However, initial symptoms such as upper-abdominal pain, nausea, and vomiting are often misdiagnosed as mild and non-specific symptoms during the antenatal or postnatal period. In the case of pregnant women, it is difficult to diagnose maternal $\mathrm{BH}$ due to a particular unique disease entity and various symptoms related to pregnancy.

Therefore, maternal BH should be suspected when dyspepsia, postprandial vomiting, epigastric pain, and hematemesis do not disappear in the last stages of pregnancy.

The frequency of herniated organs in this review is as follows: colon (70\%: Transverse $(37 \%)$, ascending $(7 \%)$, and undesignated $(26 \%))$, stomach $(63 \%)$, small intestine $(33 \%)$, spleen $(21 \%)$, pancreas $(9 \%)$, appendix $(7 \%)$, cecum $(7 \%)$, kidney $(2 \%)$, liver $(2 \%)$, and omentum $(26 \%)$. The number of herniated organs was one organ $(21 \%)$, two or three 
organs $(42 \%)$, or more than three organs $(33 \%)$, and undesignated $(4 \%)$. The most frequent single-organ occurrence was in the stomach.

\subsection{Strangulation}

Strangulation occurs when a hollow organ, the vessel etc. has become tightly constricted, such that the flow of blood or air is blocked. The following finding suggests the diagnosis of strangulated BH: (1) signs of acute gastro-intestinal obstruction, (2) cardiac shift to the right or the left side, (3) bloody fluid on aspiration of the thoracic cavity, (4) radiographic finding of diaphragm higher than the contralateral diaphragm.

Strangulation has been recognized as severe diaphragmatic hernia complications. Strangulation of diaphragmatic hernia complicating the puerperium is very rare, especially obstruction of or gangrene in the herniated viscera due to strangulated diaphragmatic hernia which is a life-threatening emergency accompanying fetal-neonatal and maternal death.

Hedblom, in 1931, revealed that mortality doubled due to intestinal obstruction during a diaphragmatic hernia. The mortality rate was $53.1 \%$ in 126 cases with obstruction and $23.8 \%$ in 252 cases without obstruction [2].

Pearson et al., in 1953, analyzed four cases of their own and 70 cases from the literature between 1798 and 1952. The mortality rate was $52.7 \%$ in 74 cases, with an operative mortality of $32.6 \%$ and non-operative mortality of $100 \%$ [59].

In this review, 44\% (19/43) of cases demonstrated signs of bowel obstruction, ischemia, or perforation of strangulated viscera during surgery, resulting in maternal death in $11 \%$ $(2 / 19)$ of cases and fetal/neonatal deaths in $21 \%(4 / 19)$ of cases (two stillborns and two neonatal deaths).

\subsection{Diagnosis}

In pregnant women with $\mathrm{BH}$, accurate diagnosis is particularly important because treatment decisions based on the diagnosis are related to life-threatening maternal and fetal events if surgical treatment is not performed at an optimal time.

Chest radiographs, ultrasonography (USG) [25], and magnetic resonance imaging (MRI) have reliable diagnostic significance for evaluating a suspected $\mathrm{BH}$ regardless of pregnancy status $[21,32,33,38,39,42]$.

A BH should be suspected if the following chest radiographic features appear: displacement of the heart across the mediastinum to the opposite side of herniated viscera, air bubbles above the diaphragm level, very high position to the contralateral side, and an opacified hemithorax, which is evidence of fluid in the chest cavity. However, it can be difficult to diagnose $\mathrm{BH}$ only with chest radiographic examination. Thus we should be considered that this diagnosis cannot be completely ruled out this diagnosis even if previous chest radiographs are a normal finding.

Ultrasonography has been recognized as the screening modality in obstetric imaging due to the advantage of easy accessibility and real-time scanning.

Ultrasonography features include a fragmented diaphragm, inability to identify the liver, spleen, kidney, the superior mesenteric and portal vessels within the normal position in the abdomen, displacement of the heart across the mediastinum, and the identification of bowel and liver in the chest. Direct visualization and detailed evaluation of the herniated viscera are crucial for accurate diagnosis and treatment.

Ultrasonography is insufficient for an adequate diagnosis. Therefore, computed tomography (CT) and MRI are useful for evaluating detailed information in pregnant patients. The exposure effects of radiation on the developing conceptus or perinatal period are related to spontaneous abortion and fetal growth restriction in the first trimester and slightly increased childhood leukemia and childhood cancer risk in the second trimester or more. In general, the use of CT during pregnancy should be avoided.

The National Council on Radiation Protection and Measurements has provided guidelines on radiation doses to ensure that a diagnostic $\mathrm{CT}$ conducted during preg- 
nancy is safe for the fetal well-being. These guidelines consider $\leq 5$ rads ( $0.5 \mathrm{~Gy}$ ) to be negligible compared to the other risks of pregnancy. Fetal radiation exposure from an abdominal/pelvis CT is $2.5 \mathrm{rad}$ ( $25 \mathrm{mGy}$ ). CT can evaluate the maternal abdomen by performing a relatively high pitch and relatively thick slice $(7-10 \mathrm{~mm})$ to minimize any risk $[22,28,30,31,34,36,40,41,43,45,60]$, but imaging should be performed only if the benefits of diagnosis exceed the theoretical risk of fetal exposure.

MRI in pregnant women may be performed on a $1.5 \mathrm{~T}$ or $3 \mathrm{~T}$ MRI scanner without the administration of an intravenous gadolinium-based contrast agent. The multiparametric imaging sequence parameters include multiplanar SPIR (spectral pre-saturation with inversion recovery) fat-suppressed T1-weighted (T1W) imaging, T2-weighted (T2W) singleshot turbo spin-echo (SSH-TSE) imaging, SPAIR (spectral attenuated inversion recovery), fat-suppressed T2W SSH-TSE imaging, and diffusion-weighted imaging (DWI).

However, the important advantage of MRI is its demonstration of a better depiction of soft-tissue contrast. MRI can be useful for evaluating abdominal pain in the pregnant patient, such as adnexal torsion, appendicitis, uterine rupture, pelvic vein thrombosis, biliary disease, and small bowel obstruction that complicate pregnancy and using MRI as the first-line modality without USG can be against the recommendation.

In strangulated herniated viscera with obstruction or ischemia, a high signal on T1W, $\mathrm{T} 2 \mathrm{~W}$, and T1W fat-saturated sequences indicates ischemia infarction. DWI can also be used as the imaging modality and is the best for describing early ischemia. Some authors have postulated that $\mathrm{T} 2 \mathrm{~W}$ contrast information of the strangulated stroma may be useful for predicting the severity of ischemic infarction.

Identification of the strangulated herniated viscera with obstruction or ischemia is important because it is necessary to determine the conversion through an abdominal approach in patients considering thoracic repair. We suggest that MRI be used as the first-line modality in pregnant women with a BH considering surgery.

\subsection{Management}

4.6.1. Guidelines for Treatment during Pregnancy

In particular, a diaphragmatic hernia during pregnancy should be treated according to the clinical picture of the gestation age in which the disease is diagnosed.

In an asymptomatic pregnant woman with a previously recognized $\mathrm{CDH}$, the choice between conservative or surgical treatment is controversial.

Given the reason for selection bias due to the disease's rarity and that most symptomatic patients had been multiparous women who experienced normal childbirth, an argument was opposed to routine repair for an asymptomatic pregnant patient with a previously recognized $\mathrm{CDH}$, even though pregnancy can trigger worsening symptoms of an unrecognized hernia,

Most of the authors reviewed by Brown agreed with the surgical indication for Bochdalek hernias in adults, suggesting that it also includes those diagnosed incidentally in radiological images.

For maternal BH during pregnancy, most authors, including Kurzel et al. [52] claim that defects in the first or second trimester should be repaired under elective conditions after administering antenatal corticosteroids before labor, regardless of the presence or absence of symptoms. In the third trimester, asymptomatic patients should undergo elective cesarean section and hernia repair simultaneously after close observation until fetal maturity.

The reasons for the routine surgical repair for the maternal BH complicating pregnancy are as follows: (i) these hernia defects may easily manifest symptoms during labor or the early postpartum period, but can manifest symptoms in the 2nd or 3rd trimester without labor; (ii) delays in treating abdominal viscera's incarceration will lead to the greater visceral displacement into the thorax by the enlarging uterus; (iii) in the later stages of pregnancy, these viscera have lost the "right of domain," and operative closure may be extremely difficult. 
In symptomatic pregnant women with a strangulated maternal $\mathrm{BH}$, or at any time during observation of visceral strangulation and obstruction, emergency surgery should be performed regardless of gestational age and fetal maturity. Further delay may result in ischemia, gangrene, or perforation.

However, some authors, including Genc and Fleyfel [21,61] suggest that gastric decompression of the nasogastric tube might improve the bowel obstruction symptoms caused by herniation. It is crucial to consider the time until surgery if antenatal corticosteroids to enhance fetal lung maturity are to be administered before transfer to a tertiary center. However, such an improvement under close monitoring of fetal and maternal vital signs is allowed for a delayed surgery.

\subsubsection{Surgical Management}

Since fetal outcomes are directly related to maternal conditions, optimal surgical intervention in pregnant women is as important as diagnosing without delay maternal and fetal well-being. Although treatment for pregnant women should be the same as that for non-pregnant women, the surgeon should operate in close relationships with obstetricians for cardiotocographic fetal monitoring before or during an operation.

It is still debatable whether the abdominal or thoracic approach and laparoscopy or laparotomy are best. Because surgeon need to find an excellent way to access the diaphragm reducing the modality during surgery.

Proponents of the abdominal approach think it offers a better chance of evaluating all abdominal organs and of treating a strangulated or perforated bowels or a volvulus. If malrotation of herniated organs is identified, repair through an abdominal approach should be implemented rather than thoracic repair. In an emergency abdominal surgery of a maternal $\mathrm{BH}$, a laparotomy provides good organ access and a good view of the surgical field, and permits efficient repair of the defect. This review demonstrated that surgery for patients with bowel obstruction, ischemia, or perforated strangulated viscera resulted in a maternal mortality rate of $11 \%$ because of sepsis and multisystem organ failure, while fetal loss was $21 \%$. Maternal $\mathrm{BH}$ with strangulated viscera should be performed before the onset of perforation or bowel necrosis.

Abdominal skin incisions are often determined by associated conditions and can be midline, paramedian, or subcostal. The surgeon should plan the incision based on the operative exposure desired to complete the procedure safely.

Types of abdominal skin incisions are often determined by the associated conditions and have included midline, paramedian, and subcostal incisions. The surgeon should plan the incision based on the operative exposure desired to complete the procedure safely. There is no evidence yet that which skin incision method is preferred or better. Securing visibility during surgery is the most important.

During surgery, a subcostal abdominal incision is a preferred approach in posterolateral $\mathrm{BH}$. This incision had the advantage of being extended across the midline with or without a vertical upper midline extension to achieve wide exposure. This incision is a preferred approach in posterolateral $\mathrm{BH}$.

Regardless of the surgical approach, there are four important steps to repairing maternal BH: reducing the hernia contents; lysing the adhesion; reconstructing the herniated viscera after complete excision of the hernia sac from the posterior pleural cavity; and repairing the hernia defect.

Primary closure repair for the herniated defect is the best treatment. Closure with excessive tension must be avoided to prevent hernia recurrence. If a tension-free closure due to large defects is not possible, the surgical technique of flaps was inevitably performed in the past. Due to tremendous advances in prosthetic material over recent decades, the surgeon can use prosthetic material that has the advantages of reducing operation time and a tension-free repair [15,26-28,30,32,33,36,38].

Kurzel, in 1988, reported the first hernia repair using synthetic polypropylene mesh in maternal BH during pregnancy. Prosthetic material for hernia repair consists of (a) synthetic 
mesh with different polymers, (b) biological mesh with the regenerative extracellular matrix, (c) composite mesh with two different surfaces, and (d) recently, drug-loaded mesh.

Despite the development of novel meshes through new technologies, recent mesh still remains a challenge to overcome postoperative complications and hernia recurrence.

There was the first hernia repair of maternal $\mathrm{BH}$ during pregnancy through the thoracoscopic approach by Julien in 2011 [32] and laparoscopic approach by Brusciano in 2003 [60].

Maternal BH with strangulated viscera should be performed before perforation or bowel necrosis occur. With the advancement of modern surgical techniques, if the hernia defect size is small and asymptomatic, it can be achieved primarily with minimally invasive surgery.

Some authors argue that the emergence of minimally invasive surgical techniques such as thoracoscopy via the thoracic approach (i.e., video-assisted thoracoscopic surgery, VATS) had decreased postoperative pain, short hospitalization compared to thoracotomy and laparotomy. For maternal $\mathrm{BH}$ cases with a stable condition during pregnancy that does not require laparotomy, if there are findings such as abnormal chest radiograph, related abdominal injury, or a right-sided defect, VATS can be recommended.

VATS can quickly and easily repair the diaphragm defect after adhesiolysis of the strangulated herniated visceral organ from the pleural cavity. There are running or interrupted sutures in the method of repair. The diaphragm repair should be performed using the interrupted suturing technique of polypropylene instead of the continuous running suturing technique.

\subsubsection{Maternal Expectant Management after Primary Surgical Intervention}

The other important point is expectant treatment until pregnancy termination after primary emergent or elective surgical intervention.

It is important to prevent preterm labor or preterm birth during maternal expectant management until delivery after surgery. Studies have demonstrated that preterm labor by non-obstetric elective surgical intervention accounts for $5 \%$ of cases.

According to trimester, our results of treatment showed 16 cases underwent delayed delivery after surgical repair with diagnosing hernia. Of 11 cases (69\%) in the 1st and 2nd trimester, 10 surgeries were immediately performed, and 1 surgical repair after expectant management was attempted. The duration of expectant management until delivery after hernia repair surgery in this period ranged from 5 days to 13 weeks.

In five cases (31\%) in the 3rd trimester, three immediate surgery and two surgical repairs with expectant management were performed. The duration of follow-up until delivery after hernia repair surgery ranged from 3 days to 10 weeks.

Ten cases underwent simultaneous delivery with surgical repair. Of two cases $(22 \%)$ in the 1st and 2nd trimesters, one surgery was immediately performed, and one surgical repair after expectant management was attempted. In seven cases (82\%) in the 3rd trimester, four immediate surgery and three surgical repairs with expectant management were performed. In one case in the postpartum, one surgery was immediately performed. The duration of follow-up until delivery after hernia repair surgery ranged from 7 days to 3 weeks.

Tocolytics (i.e., terbutaline, magnesium) used for postoperative preterm labor is generally well controlled in the patient with elective surgical intervention. However, preterm delivery may be uncontrolled in some patients with emergent surgical intervention. Gestational age at treatment and severity of the underlying disease is the most predictive indicator of patients at risk for preterm delivery. The risk of preterm delivery is higher in the later trimester and for severe $\mathrm{BH}$.

During expectant management, it is important to achieve appropriate nutritional support. Because malnutrition for this period can be associated with maternal and fetal morbidity, including fetal growth restriction, maternal weight loss, and electrolyte imbalances, therefore, specialized nutritional support can be delivered enterally, usually via 
nasogastric tube feeding, feeding jejunostomy, or parenteral feeding via intravenous vessels with peripheral or central venous access

\subsection{Strengths and Limitations}

This study's main strength is that both maternal and fetal outcomes related to maternal $\mathrm{BH}$ were documented. Until now, there had been limited attempts to systematically review the outcome data during pregnancy.

There has been no randomized study due to the rarity of the condition. Therefore, a meta-analysis, including the multiplicity of confounding factors influencing the outcome, could not be performed. Under such a circumstance, an evidence-based survey by case series or case report was adopted as the only way to conduct a systematic review, which identified all eligible studies through detailed data extraction.

First, this review was affected by selection bias because of the limited data available for maternal $\mathrm{BH}$, as well as publication bias. Second, all patients under review were classified as having maternal $\mathrm{BH}$ according to the inclusion criteria. However, it may be difficult to determine whether the hernia could be classified as a preexisting congenital type due to developmental disabilities in the embryonic period, an acquired type due to gradual irritational pressure, or sudden trauma, or even a mixed type.

\section{Conclusions}

Early diagnosis and definitive surgical intervention are imperative, as gangrenous or a non-viable bowel resection significantly increase mortality. Therefore, multidisciplinary care should be required for maternal BH cases who undergo surgical repair during pregnancy, and the mother and fetus should receive individualized care for optimal results.

Supplementary Materials: The following are available online at https:/ / www.mdpi.com/article/10 .3390 / diagnostics11071261/s1, TableS1: Summary of the 94 studies included in the search review; Table S2: Final summary of the 42 studies.

Author Contributions: Conceptualization, J.-Y.C. and S.-H.L.; methodology, J.-Y.C. and S.-H.L.; software, J.-H.L. and S.-H.L.; validation, H.-J.R., S.-J.L., and J.-W.A.; formal analysis, J.-Y.C. and S.-H.L.; investigation, J.-H.L., J.-W.A., and J.-Y.C.; resources, H.-J.R. and J.-H.L.; data curation, J.-Y.C. and J.-S.K.; writing—original draft preparation, S.-H.L.; writing—review and editing, S.-H.L.; visualization, S.-H.L.; supervision, S.-H.L.; project administration, S.-S.Y. and S.-H.L. All authors read and agreed to the published version of the manuscript.

Funding: This research received no external funding.

Data Availability Statement: The data presented in this study are available upon request.

Conflicts of Interest: The authors declare no conflict of interest.

\section{References}

1. McGivern, M.R.; Best, K.E.; Rankin, J.; Wellesley, D.; Greenlees, R.; Addor, M.C.; Arriola, L.; de Walle, H.; Barisic, I.; Beres, J.; et al. Epidemiology of congenital diaphragmatic hernia in Europe: A register-based study. Arch. Dis. Child. Fetal Neonatal Ed. 2015, 100, F137-F144. [CrossRef]

2. Hedblom, C.A. Diphragmatic hernia. A study of three hundred and seventy eight cases in which operation was performed. JAMA 1925, 85, 947-953. [CrossRef]

3. Mullins, M.E.; Stein, J.; Saini, S.S.; Mueller, P.R. Prevalence of incidental Bochdalek's hernia in a large adult population. Am. J. Roentgenol. 2001, 177, 363-366. [CrossRef] [PubMed]

4. Thompson, J.; Le Blanc, L.J. Congenital diaphragmatic hernia: Visceral strangulation complicating delivery. Am. J. Surg. 1945, 67, 123-130. [CrossRef]

5. Pearson, S.C.; Pillsbury, S.G.; Mccallum, M. Strangulated diaphragmatic hernia complicating delivery. J. Am. Med Assoc. 1950, 144, 22-24. [CrossRef]

6. Murless, B.C. Hernia of the diaphragm as a complication of labour. Br. Med. J. 1947, 2, 251. [CrossRef]

7. Hodge, K. Diaphragmatic hernia complicated by gastric ulcer and pregnancy. Br. J. Radiol. 1950, 23, 573. [CrossRef] [PubMed]

8. Hushlan, S. Diaphragmatic hernia in pregnancy; significance and danger. Conn. State Med J. 1951, 15, 969.

9. Osborne, W.; Foster, C. Diaphragmatic hernia complicating pregnancy. Am. J. Obstet. Gynecol. 1953, 66, 682-684. [CrossRef] 
10. Hobbins, W.; Hurwitz, C. Incarcerated diaphragmatic hernia of the colon occurring during pregnancy. New Engl. J. Med. 1953, 249, 773-774. [CrossRef] [PubMed]

11. Flood, J.L. Foramen of Bochdalek hernia in pregnancy. J. Indiana State Med. Assoc. 1963, 56, 32-34. [PubMed]

12. Savage, P.T. Obstructed volvulus of the stomach in a diaphragmatic hernia: A post-partum emergency. Proc. R Soc. Med. 1968, 61, 956-957. [PubMed]

13. Gimovsky, M.; Schifrin, B. Incarcerated foramen of Bochdalek hernia during pregnancy. A case report. J. Reprod. Med. 1983, 28, 156-158.

14. Reed, M.R.; De Silva, P.D.; Mostafa, S.; Collins, F. Diaphragmatic hernia in pregnancy. Br. J. Surg. 1987, 74. [CrossRef]

15. Kurzel, R.B.; Naunheim, K.S.; Schwartz, R.A. Repair of symptomatic diaphragmatic hernia during pregnancy. Obstet. Gynecol. 1988, 71, 869-871.

16. Toorians, A.W.; Drost-Driessen, M.A.; Snellen, J.P.; Smeets, R.W. Acute hernia of Bochdalek during pregnancy Hyperemesis for the first time in a third pregnancy? Acta Obstet. Gynecol. Scand. 1992, 71, 547-549. [CrossRef]

17. Hill, R.; Heller, M.B. Diaphragmatic rupture complicating labor. Ann. Emerg. Med. 1996, 27, 522-524. [CrossRef]

18. Ortega-Carnicer, J.; Ambros, A.; Alcazar, R. Obstructive shock due to labor-related diaphragmatic hernia. Crit. Care Med. 1998, 26, 616-618. [CrossRef] [PubMed]

19. Seon Cha, A.; Krew, M.A.; Tamlyn, T.; Gill, P. Gastric rupture associated with pregnancy. Obstet. Gynecol. 2002, 100, 1072-1074. [CrossRef]

20. Williams, M.; Appelboam, R.; McQuillan, P. Presentation of diaphragmatic herniae during pregnancy and labour. Int. J. Obstet. Anesth. 2003, 12, 130-134. [CrossRef]

21. Genc, M.R.; Clancy, T.E.; Ferzoco, S.J.; Norwitz, E. Maternal congenital diaphragmatic hernia complicating pregnancy. Obstet. Gynecol. 2003, 102, 1194-1196.

22. Luu, T.D.; Reddy, V.S.; Miller, D.L.; Force, S.D. Gastric rupture associated with diaphragmatic hernia during pregnancy. Ann. Thorac. Surg. 2006, 82, 1908-1910. [CrossRef]

23. Eglinton, T.W.; Coulter, G.N.; Bagshaw, P.F.; Cross, L.A. Diaphragmatic hernias complicating pregnancy. ANZ J. Surg. 2006, 76, 553-557. [CrossRef] [PubMed]

24. Barbetakis, N.; Efstathiou, A.; Vassiliadis, M.; Xenikakis, T.; Fessatidis, I. Bochdaleck's hernia complicating pregnancy: Case report. World J. Gastroenterol. WJG 2006, 12, 2469. [CrossRef]

25. Rajasingam, D.; Kakarla, A.; Jones, A.; Ash, A. Strangulated congenital diaphragmatic hernia with partial gastric necrosis: A rare cause of abdominal pain in pregnancy. Int. J. Clin. Pract. 2007, 61, 1587-1589. [CrossRef] [PubMed]

26. Pai, S.; Balu, K.; Madhusudhanan, J. Congenital diaphragmatic hernia complicating pregnancy: A case report. Internet J. Gynecol. Obstet. 2007, 9.

27. Palanivelu, C.; Rangarajan, M.; Maheshkumaar, G.; Parthasarathi, R. Laparoscopic mesh repair of a Bochdalek diaphragmatic hernia with acute gastric volvulus in a pregnant patient. Singap. Med. J. 2008, 49, e26-e28.

28. Sano, A.; Kato, H.; Hamatani, H.; Sakai, M.; Tanaka, N.; Inose, T.; Kimura, H.; Kuwano, H. Diaphragmatic hernia with ischemic bowel obstruction in pregnancy: Report of a case. Surg. Today 2008, 38, 836. [CrossRef]

29. Hunter, J.D.; Nimmagadda, J.; Quayle, A. Maternal congenital diaphragmatic hernia causing cardiovascular collapse during pregnancy. Br. J. Hosp. Med. 2009, 70, 166-167. [CrossRef]

30. Islah, M.; Jiffre, D. A rare case of incarcerated bochdalek diaphragmatic hernia in a pregnant lady. Med J. Malays. 2010, 65, 75-76.

31. Morcillo-López, I.; Hidalgo-Mora, J.J.; Baamonde, A.; Díaz-García, C. Gastric and diaphragmatic rupture in early pregnancy. Interact. Cardiovasc. Thorac. Surg. 2010, 11, 713-715. [CrossRef] [PubMed]

32. Julien, F.; Drolet, S.; Lévesque, I.; Bouchard, A. The right lateral position for laparoscopic diaphragmatic hernia repair in pregnancy: Technique and review of the literature. J. Laparoendosc. Adv. Surg. Tech. A 2011, 21, 67-70. [CrossRef]

33. Ngai, I.; Sheen, J.-J.; Govindappagari, S.; Garry, D.J. Bochdalek hernia in pregnancy. Case Rep. 2012, 2012, bcr2012006859. [CrossRef]

34. Hamaji, M.; Burt, B.M.; Ali, S.O.; Cohen, D.M. Spontaneous diaphragm rupture associated with vaginal delivery. Gen. Thorac. Cardiovasc. Surg. 2013, 61, 473-475. [CrossRef] [PubMed]

35. Wieman, E.; Pollock, G.; Moore, B.T.; Serrone, R. Symptomatic right-sided diaphragmatic hernia in the third trimester of pregnancy. JSLS J. Soc. Laparoendosc. Surg. 2013, 17, 358. [CrossRef]

36. Ali, S.A.; Haseen, M.A.; Beg, M.H. Agenesis of right diaphragm in the adults: A diagnostic dilemma. Indian J. Chest Dis. Allied Sci. 2014, 56, 121-123. [PubMed]

37. Debergh, I.; Fierens, K. Laparoscopic repair of a Bochdalek hernia with incarcerated bowel during pregnancy: Report of a case. Surg. Today 2014, 44, 753-756. [CrossRef]

38. Hernández-Aragon, M.; Rodriguez-Lazaro, L.; Crespo-Esteras, R.; Ruiz-Campo, L.; Adiego-Calvo, I.; Campillos-Maza, J. Bochdalek diaphragmatic hernia complicating pregnancy in the third trimester: Case report. Obstet. Gynaecol. Cases Rev. 2015, 2. [CrossRef]

39. Yetkinel, S.; Pekşen, Ç.; Kızıltan, R. Symptomatic Bochdalek Hernia in Pregnancy: A Rare Case Report. Case Rep. Surg. 2017, 2017, 2862149. [CrossRef]

40. Reddy, M.; Kroushev, A.; Palmer, K. Undiagnosed maternal diaphragmatic hernia-a management dilemma. BMC Pregnancy Childbirth 2018, 18, 1-5. [CrossRef] [PubMed] 
41. Matsudera, S.; Nakajima, M.; Takahashi, M.; Muroi, H.; Kikuchi, M.; Shida, Y.; Ihara, K.; Yamaguchi, S.; Sasaki, K.; Tsuchioka, T.; et al. Laparoscopic surgery for a Bochdalek hernia triggered by pregnancy in an adult woman: A case report. Int. J. Surg. Case Rep. 2018, 48, 10-15. [CrossRef]

42. Vasquez, D.N.; Basualdo, M.N.; Aphalo, V.M.; Carreras, L.P.; Plotnikow, G.A.; Intile, A.D.; Moreira, J. Complications of congenital hernia in pregnancy: A case report. A A Pract. 2019, 13, 102-106. [CrossRef] [PubMed]

43. Suhardja, T.S.; Vaska, A.; Foley, D.; Gribbin, J. Adult Bochdalek hernia in a pregnant woman. ANZ J. Surg. 2019, 89 , E162-E163. [CrossRef] [PubMed]

44. Ménassa, M.; Bergeron, A.M.; Drolet, S.; Bouchard, A. Strangulated congenital diaphragmatic hernia of bochdalek diagnosed in late pregnancy: A case report and review of the literature. J. Obstet. Gynaecol. Can. 2019, 41, 1482-1484. [CrossRef]

45. Haj-Yahia, S.; Al Aqra, A.; Abed, K.; Bali, K.; Sbaih, M.N.; Al Asmar, M.; Caputo, M.; Othman, W.; Al-Adhami, A. Rare case of diaphragmatic rupture following resuscitation in a pregnant woman first in literature. J. Cardiothorac. Surg. 2020, 15, 44. [CrossRef]

46. Greer, J.J. Current concepts on the pathogenesis and etiology of congenital diaphragmatic hernia. Respir. Physiol. Neurobiol. 2013, 189, 232-240. [CrossRef] [PubMed]

47. Veenma, D.C.; de Klein, A.; Tibboel, D. Developmental and genetic aspects of congenital diaphragmatic hernia. Pediatr. Pulmonol. 2012, 47, 534-545. [CrossRef]

48. Pober BR: Overview of epidemiology, genetics, birth defects, and chromosome abnormalities associated with CDH. Am. J. Med. Genet. C Semin. Med. Genet. 2007, 145, 158-171.

49. Torfs, C.P.; Curry, C.J.; Bateson, T.F.; Honoré, L.H. A population-based study of congenital diaphragmatic hernia. Teratology 1992, 46, 555-565. [CrossRef] [PubMed]

50. Dott, M.M.; Wong, L.Y.; Rasmussen, S.A. Population-based study of congenital diaphragmatic hernia: Risk factors and survival in Metropolitan Atlanta, 1968-1999. Birth Defects Res. Part A Clin. Mol. Teratol. 2003, 67, 261-267. [CrossRef]

51. Brown, S.R.; Horton, J.D.; Trivette, E.; Hofmann, L.J.; Johnson, J.M. Bochdalek hernia in the adult: Demographics, presentation, and surgical management. Hernia 2011, 15, 23-30. [CrossRef]

52. Merrell, A.J.; Ellis, B.J.; Fox, Z.D.; Lawson, J.A.; Weiss, J.A.; Kardon, G. Muscle connective tissue controls development of the diaphragm and is a source of congenital diaphragmatic hernias. Nat. Genet. 2015, 47, 496-504. [CrossRef]

53. Jay, P.Y.; Bielinska, M.; Erlich, J.M.; Mannisto, S.; Pu, W.T.; Heikinheimo, M.; Wilson, D.B. Impaired mesenchymal cell function in Gata4 mutant mice leads to diaphragmatic hernias and primary lung defects. Dev. Biol. 2007, 301, 602-614. [CrossRef] [PubMed]

54. Norden, J.; Grieskamp, T.; Lausch, E.; van Wijk, B.; van den Hoff, M.J.; Englert, C.; Petry, M.; Mommersteeg, M.T.; Christoffels, V.M.; Niederreither, K.; et al. Wt1 and retinoic acid signaling in the subcoelomic mesenchyme control the development of the pleuropericardial membranes and the sinus horns. Circ. Res. 2010, 106, 1212-1220. [CrossRef] [PubMed]

55. Ijpenberg, A.; Pérez-Pomares, J.M.; Guadix, J.A.; Carmona, R.; Portillo-Sánchez, V.; Macías, D.; Hohenstein, P.; Miles, C.M.; Hastie, N.D.; Muñoz-Chápuli, R. Wt1 and retinoic acid signaling are essential for stellate cell development and liver morphogenesis. Dev. Biol. 2007, 312, 157-170. [CrossRef]

56. You, L.R.; Takamoto, N.; Yu, C.T.; Tanaka, T.; Kodama, T.; Demayo, F.J.; Tsai, S.Y.; Tsai, M.J. Mouse lacking COUP-TFII as an animal model of Bochdalek-type congenital diaphragmatic hernia. Proc. Natl. Acad. Sci. USA 2005, 102, 16351-16356. [CrossRef] [PubMed]

57. Tenbrinck, R.; Gaillard, J.L.; Tibboel, D.; Kluth, D.; Lachmann, B.; Molenaar, J.C. Pulmonary vascular abnormalities in experimentally induced congenital diaphragmatic hernia in rats. J. Pediatr. Surg. 1992, 27, 862-865. [CrossRef]

58. Greer, J.J.; Babiuk, R.P.; Thebaud, B. Etiology of congenital diaphragmatic hernia: The retinoid hypothesis. Pediatr. Res. 2003, 53, 726-730. [CrossRef]

59. Pearson, S. Strangulated diaphragmatic hernia: Report of four cases. AMA Arch. Surg. 1953, 66, 155-166. [CrossRef]

60. Brusciano, L.; Izzo, G.; Maffettone, V.; Rossetti, G.; Renzi, A.; Napolitano, V.; Russo, G.; Del Genio, A. Laparoscopic treatment of Bochdalek hernia without the use of a mesh. Surg. Endosc. 2003, 17, 1497-1498. [CrossRef] [PubMed]

61. Fleyfel, M.; Provost, N.; Ferreira, J.F.; Porte, H.; Bourzoufi, K. Management of diaphragmatic hernia during pregnancy. Anesth Analg. 1998, 86, 501-503. [PubMed] 